COMMUNICATIONS IN

ANALYSIS AND GEOMETRY

Volume 14, Number 4, 633-671, 2006

\title{
On the equivariant Gromov-Witten theory of $\mathbb{P}^{2}$-bundles over curves
}

\author{
Amin Gholampour
}

\begin{abstract}
We study equivariant Gromov-Witten invariants of $\mathbb{P}^{2}$-bundles over a curve $C$. Our bundles are of the form $\mathbb{P}\left(L_{0} \oplus L_{1} \oplus L_{2}\right)$ for arbitrary line bundles $L_{0}, L_{1}$ and $L_{2}$ over $C$. We compute the partition functions of these invariants for all classes of the form $s+n f$, where $s$ is a section, $f$ is a fiber and $n$ is an integer. In the case where the class is Calabi-Yau, i.e., $K \cdot(s+n f)=0$, the partition function is given by

$$
3^{g}\left(2 \sin \frac{u}{2}\right)^{2 g-2} .
$$

As an application, one can obtain a series of full predictions for the equivariant Donaldson-Thomas invariants for this family of non-toric three-folds.
\end{abstract}

\section{Introduction}

Let $X$ be a $\mathbb{P}^{2}$-bundle over a smooth complex projective curve $C$ of genus $g$, and let $K_{X}$ be its canonical class. We denote the cohomology class of the fiber by $F$.

Definition 1.1. A class $\beta \in H^{4}(X, \mathbb{Z})$ is called section class if $F \cdot \beta=1$. We say $\beta$ is a Calabi-Yau class if $K_{X} \cdot \beta=0$. $\beta$ is called Calabi-Yau section class if both conditions hold.

Remark 1.2. A section class is not necessarily represented by a geometric section of the bundle $X$. It could be, for example, a section with a number of fiber curves attached to it.

Now let $X$ be a $\mathbb{P}^{2}$-bundle of the form

$$
\mathbb{P}\left(L_{0} \oplus L_{1} \oplus L_{2}\right) \longrightarrow C
$$

where $C$ is a curve of genus $g$, and $L_{i} \rightarrow C$ is a line bundle of degrees $k_{i}$. Without loss of generality, we will assume that $L_{0}$ is the trivial bundle. As 
in [2], we use the word level to refer to $\left(k_{1}, k_{2}\right)$. It can be shown that the canonical class of $X$ is given by

$$
K_{X}=-3 H+\left(2 g-2-k_{1}-k_{2}\right) F
$$

where $H$ is the first Chern class of $\mathcal{O}(1) \rightarrow X$.

Definition 1.3. There is a distinguished section in $X$, which is by definition the locus of $(1: 0: 0)$ in $X$. We denote by $\beta_{0}$ the cohomology class in $H^{4}(X, \mathbb{Z})$, which is represented by this locus. We also define

$$
f:=H \cdot F \in H^{4}(X, \mathbb{Z})
$$

Note that $\left\{\beta_{0}, f\right\}$ is a set of generators for $H^{4}(X, \mathbb{Z})$ and also the relations $H \cdot \beta_{0}=0$ and $F \cdot \beta_{0}=1$ hold in the cohomology ring. The latter implies that $\beta_{0}$ is a section class.

Remark 1.4. One can see that for the $\mathbb{P}^{2}$-bundles of this form,

$$
\beta \in H^{4}(X, \mathbb{Z})
$$

is a section class (see Definition 1.1) if and only if it is of the form

$$
\beta=\beta_{0}+n f
$$

for an integer $n$ (see also Remark 1.2).

The complex torus $\mathbb{T}=\left(\mathbb{C}^{*}\right)^{3}$ acts on $X$ by

$$
\left(z_{0}, z_{1}, z_{2}\right)\left(x_{0}: x_{1}: x_{2}\right) \longmapsto\left(z_{0} x_{0}: z_{1} x_{1}: z_{2} x_{2}\right) .
$$

Let $\beta_{s} \in H^{4}(X, \mathbb{Z})$ be a section class. The partition function of the degree $\beta_{s}$ Gromov-Witten invariants is given by

$$
Z_{\beta_{s}}\left(g \mid k_{1}, k_{2}\right)=\sum_{h=0}^{\infty} u^{2 h-2-K_{X} \cdot \beta_{s}} \int_{\left[\bar{M}_{h}\left(X, \beta_{s}\right)\right]^{\mathrm{vir}}} 1,
$$

where $\bar{M}_{h}\left(X, \beta_{s}\right)$ is the moduli space of degree $\beta_{s}$, genus $h$ stable maps ${ }^{1}$ to $X$, and $\left[\bar{M}_{h}\left(X, \beta_{s}\right)\right]^{\text {vir }} \in A_{D}^{\mathbb{T}}\left(\bar{M}_{h}\left(X, \beta_{s}\right)\right)$ is in the $D$ th equivariant Chow

${ }^{1}$ We assume that all domain curves are connected (see Remark 2.4). 
group for

$$
D=-K_{X} \cdot \beta_{s}=\operatorname{virdim} \bar{M}_{h}\left(X, \beta_{s}\right) .
$$

Since we are working equivariantly (the invariants are defined by equivariantly pushing forward to a point), our definition makes sense even for negative values of $D$ (c.f. Section 2.1 of [2]).

Remark 1.5. The equivariant Gromov-Witten partition functions are invariant under equivariant deformations. The space $X$ that we work with is determined up to equivariant deformation by $g$, the genus of $C$, and the level $\left(k_{1}, k_{2}\right)$, and so in this paper we can refer to $X$ by specifying only these parameters.

Let $t_{0}, t_{1}, t_{2}$ be the generators for the equivariant Chow group of a point:

$$
A_{*}^{\mathbb{T}}(p t) \cong \mathbb{Q}\left[t_{0}, t_{1}, t_{2}\right]
$$

$Z_{\beta_{s}}\left(g \mid k_{1}, k_{2}\right)$ is a homogeneous polynomial in $t_{0}, t_{1}, t_{2}$ of degree $-D$ with coefficients in $\mathbb{Q}((u))$. In particular, it is zero if $D$ is positive, and it is a Laurent series in $u$, independent of $t_{0}, t_{1}, t_{2}$, when $D=0$ (this happens when $\beta_{s}$ is a Calabi-Yau section class). In the later case, $Z_{\beta_{s}}\left(g \mid k_{1}, k_{2}\right)$ is equal to the usual Gromov-Witten partition function. (c.f. Section 2.1 of [2]).

The partition function of the section class Gromov-Witten invariants is given by:

$$
Z\left(g \mid k_{1}, k_{2}\right)=\sum_{\beta_{s} \text { is a section class }} Z_{\beta_{s}}\left(g \mid k_{1}, k_{2}\right) .
$$

We will define the relative version of $Z\left(g \mid k_{1}, k_{2}\right)$ and prove a gluing theorem for it. The gluing formula allows us to compute the partition function in the general case in terms of the basic partition functions for the case of $g=0$, relative to one, two or three fibers. We will compute these basic partition functions via localization techniques combined with relations arising from the gluing formula. These give rise to explicit $3 \times 3$ matrices $G, U_{1}$ and $U_{2}$ with entries in $\mathbb{Q}((u))\left(t_{0}, t_{1}, t_{2}\right)$. We will then prove the main result of this paper, which gives a formula for $Z\left(g \mid k_{1}, k_{2}\right)$ for any given genus $g$ and level $\left(k_{1}, k_{2}\right)$.

Theorem 1.6. Let $X$ be a $\mathbb{P}^{2}$-bundle over a curve $C$ of genus $g$ of the form $\mathbb{P}\left(\mathcal{O} \oplus L_{1} \oplus L_{2}\right)$, where $L_{1}$ and $L_{2}$ are two line bundles of degrees $k_{1}$ and $k_{2}$, 
respectively. Then

$$
Z\left(g \mid k_{1}, k_{2}\right)=\operatorname{tr}\left(G^{g-1} U_{1}^{k_{1}} U_{2}^{k_{2}}\right)
$$

where $G, U_{1}$ and $U_{2}$ are given by

$$
\begin{aligned}
& G=\left[\begin{array}{ccc}
\left(t_{0}-t_{1}\right)\left(t_{0}-t_{2}\right) & 0 & 0 \\
0 & \left(t_{1}-t_{0}\right)\left(t_{1}-t_{2}\right) & 0 \\
0 & 0 & \left(t_{2}-t_{0}\right)\left(t_{2}-t_{1}\right)
\end{array}\right] \\
& +\left[\begin{array}{ccc}
\frac{2\left(2 t_{0}-t_{1}-t_{2}\right)}{\left(t_{0}-t_{1}\right)\left(t_{0}-t_{2}\right)} & \frac{t_{0}+t_{1}-2 t_{2}}{\left(t_{0}-t_{1}\right)\left(t_{0}-t_{2}\right)} & \frac{t_{0}+t_{2}-2 t_{1}}{\left(t_{0}-t_{1}\right)\left(t_{0}-t_{2}\right)} \\
\frac{t_{0}+t_{1}-2 t_{2}}{\left(t_{1}-t_{0}\right)\left(t_{1}-t_{2}\right)} & \frac{2\left(2 t_{1}-t_{0}-t_{2}\right)}{\left(t_{1}-t_{0}\right)\left(t_{1}-t_{2}\right)} & \frac{t_{1}+t_{2}-2 t_{0}}{\left(t_{1}-t_{0}\right)\left(t_{1}-t_{2}\right)} \\
\frac{t_{0}+t_{2}-2 t_{1}}{\left(t_{2}-t_{0}\right)\left(t_{2}-t_{1}\right)} & \frac{t_{1}+t_{2}-2 t_{0}}{\left(t_{2}-t_{0}\right)\left(t_{2}-t_{1}\right)} & \frac{2\left(2 t_{2}-t_{0}-t_{1}\right)}{\left(t_{2}-t_{0}\right)\left(t_{2}-t_{1}\right)}
\end{array}\right] \phi^{3}, \\
& U_{1}=\left[\begin{array}{ccc}
\frac{\phi}{t_{0}-t_{1}} & \frac{\left(t_{1}-t_{2}\right) \phi}{\left(t_{0}-t_{1}\right)\left(t_{0}-t_{2}\right)} & 0 \\
\frac{\phi}{t_{1}-t_{0}} & \frac{\left(t_{1}-t_{0}\right)^{2}\left(t_{1}-t_{2}\right)^{2} \phi^{-2}+\left(2 t_{1}-t_{0}-t_{2}\right) \phi}{\left(t_{1}-t_{0}\right)\left(t_{1}-t_{2}\right)} & \frac{\phi}{t_{1}-t_{2}} \\
0 & \frac{\left(t_{1}-t_{0}\right) \phi}{\left(t_{2}-t_{0}\right)\left(t_{2}-t_{1}\right)} & \frac{\phi}{t_{2}-t_{1}}
\end{array}\right], \\
& U_{2}=\left[\begin{array}{ccc}
\frac{\phi}{t_{0}-t_{2}} & 0 & \frac{\left(t_{2}-t_{1}\right) \phi}{\left(t_{0}-t_{1}\right)\left(t_{0}-t_{2}\right)} \\
0 & \frac{\phi}{t_{1}-t_{2}} & \frac{\left(t_{2}-t_{0}\right) \phi}{\left(t_{1}-t_{0}\right)\left(t_{1}-t_{2}\right)} \\
\frac{\phi}{t_{2}-t_{0}} & \frac{\phi}{t_{2}-t_{1}} & \frac{\left(t_{2}-t_{0}\right)^{2}\left(t_{2}-t_{1}\right)^{2} \phi^{-2}+\left(2 t_{2}-t_{0}-t_{1}\right) \phi}{\left(t_{2}-t_{0}\right)\left(t_{2}-t_{1}\right)}
\end{array}\right] \text {, }
\end{aligned}
$$

where $\phi=2 \sin \frac{u}{2}$.

As an application of Theorem 1.6, we prove the following result:

Theorem 1.7. Let $X$ be any $\mathbb{P}^{2}$-bundle over a curve $C$ of genus $g$, and let $\beta_{c s} \in H^{4}(X, \mathbb{Z})$ be a Calabi-Yau section class, Then

$$
Z_{\beta_{c s}}(g)=3^{g}\left(2 \sin \frac{u}{2}\right)^{2 g-2}
$$


where $Z_{\beta_{c s}}(g)$ is the usual partition function for the degree $\beta_{c s}$ GromovWitten invariants of $X$, given by

$$
Z_{\beta_{c s}}(g)=\sum_{h=0}^{\infty} u^{2 h-2} \int_{\left[\bar{M}_{h}\left(X, \beta_{c s}\right)\right]^{\mathrm{vir}}} 1 .
$$

\subsection{Donaldson-Thomas invariant prediction}

Another application of Theorem 1.6 is the prediction for equivariant Donaldson-Thomas partition functions (see Section 9 of [2] and also see $[9,10])$.

As an example, by the assumptions of Theorem 1.7, and taking $e^{i u}=-q$, we can write

$$
Z_{\beta_{c s}}^{\mathrm{DT}}(g)=3\left[3\left(q+q^{-1}+2\right) M(-q)^{18}\right]^{g-1},
$$

where

$$
Z_{\beta_{c s} \mathrm{DT}}^{\mathrm{D}}(g)=\sum_{n \in \mathbb{Z}} q^{n} \int_{\left[I_{n}\left(X, \beta_{c s}\right)\right]^{\mathrm{vir}}} 1,
$$

and

$$
M(q)=\prod_{j \geq 1}\left(1-q^{j}\right)^{-j}
$$

Moreover, by the same notations as in Theorem 1.6, if we define the partition function for the class $\beta_{s}$ equivariant Donalsdson-Thomas invariants as

$$
Z_{\beta_{s}}^{\mathrm{DT}}\left(g \mid k_{1}, k_{2}\right)=(-q)^{-(1 / 2) K_{X} \cdot \beta_{s}} \sum_{n \in \mathbb{Z}} q^{n} \int_{\left[I_{n}\left(X, \beta_{s}\right)\right]^{\mathrm{vir}}} 1
$$

then Theorem 1.6 gives the full prediction for $Z_{\beta_{s}}^{\mathrm{DT}}\left(g \mid k_{1}, k_{2}\right)$ for any section class $\beta_{s}$.

The GW/DT correspondence in the case $\beta_{s}=\beta+f$ has recently been worked out to the first order in [3]. See also Appendix B for more explicit formulas on the Gromov-Witten theory side.

\subsection{Plan of the paper}

In Section 2, we define the partition function of the relative Gromov-Witten invariants of the space $\mathbb{P}\left(\mathcal{O} \oplus L_{1} \oplus L_{2}\right)$. Then we express a gluing theorem for these partition functions. 
In Section 3, we compute some of the basic partition functions we defined in Section 2 in the case $g=0$. There are some basic partition functions in this case that we can compute via localization, we compute them in 3.1. We use the gluing theorem of Section 2 to compute those that we cannot compute via localization. This will be done in Section 3.3.

In Section 4, using the results of Section 3, we construct the matrices $G, U_{1}$ and $U_{2}$, which appeared in Theorem 1.6 and then we prove Theorem 1.6 .

In Section 5, we first prove (Lemma 5.1) that any $\mathbb{P}^{2}$-bundle over a curve $C$ is deformation equivalent to a $\mathbb{P}^{2}$-bundle over $C$ of the form $\mathbb{P}(\mathcal{O} \oplus \mathcal{O} \oplus L)$. Having this, we use Theorem 1.6 to prove Theorem 1.7.

In Appendix A, we first prove that it is enough in this paper to only consider the moduli space of maps with connected domains (Lemma A.1). After that we give a proof for the gluing theorem expressed in Section 2.

In Appendix B, we provide some more formulas for the partition function of equivariant Gromov-Witten invariants in some special cases.

\section{Relative invariants and the Gluing theorem}

Let $\left(C, p_{1}, \ldots, p_{r}\right)$ be a non-singular curve of genus $g$ with $r$ marked points. Following the notations of Section 1, we take

$$
X=\mathbb{P}\left(\mathcal{O} \oplus L_{1} \oplus L_{2}\right) \longrightarrow\left(C, p_{1}, \ldots, p_{r}\right)
$$

We will review the definition of the section class equivariant Gromov-Witten invariants relative to divisors $F_{1}, \ldots, F_{r}$, where $F_{i}$ is the fiber over the point $p_{i}$. For a treatment of the foundations of equivariant relative GromovWitten theory, see [5].

The complex torus $\mathbb{T}=\left(\mathbb{C}^{*}\right)^{3}$ acts on $X$ as in Section 1 . We need to fix a basis, $\mathcal{B}_{p}$, for the equivariant Chow group of each fiber, $F_{p}$, which is a copy of $\mathbb{P}^{2}$ :

$$
A_{*}^{\mathbb{T}}\left(F_{p}\right) \cong A_{*}^{\mathbb{T}}\left(\mathbb{P}^{2}\right) \cong \mathbb{Z}\left[H, t_{0}, t_{1}, t_{2}\right] /\left(\prod_{j=0}^{2}\left(H-t_{j}\right)\right)
$$

Let $\beta_{s} \in H^{4}(X, \mathbb{Z})$ be a section class (defined in Section 1 ). We take

$$
Z_{\beta_{s}}^{h}\left(g \mid k_{1}, k_{2}\right)_{\alpha_{1} \cdots \alpha_{r}}
$$


to be degree $\beta_{s}$, genus $h$, equivariant Gromov-Witten invariant of $X$ relative to the divisors $F_{1}, \ldots, F_{r}$, with restrictions given by $\alpha_{p} \in \mathcal{B}_{p}$, one for each divisor. More precisely, we take

$$
\vec{L}=\left(l_{1}, \ldots, l_{r}\right) \in\left(\mathbb{Z}^{+}\right)^{r}, \quad \vec{F}=\left(F_{1}, \ldots, F_{r}\right) .
$$

Then following Section 2 of [10], let $X[\vec{L}]$ be the $l_{p}$-step degeneration of $X$ along each $F_{p}$, and let $\bar{M}_{h}\left(X / \vec{F}, \beta_{s}\right)$ be the moduli space of relative stable maps $q: C^{\prime} \rightarrow X[\vec{L}]$ from nodal genus $h$ curves $^{2}, C^{\prime}$, to $X[\vec{L}]$, for some $\vec{L}$, which are representing the class $\beta_{s}$. Then, $\bar{M}_{h}\left(X / \vec{F}, \beta_{s}\right)$ is a DM-stack of virtual dimension $-K_{X} \cdot \beta_{s}$ (see also [7]).

For each $p=1, \ldots, r$, we have an evaluation map which is determined by relative points and is $\mathbb{T}$-equivariant (see [8]):

$$
\mathrm{ev}_{p}: \bar{M}_{h}\left(X / \vec{F}, \beta_{s}\right) \longrightarrow F_{p} .
$$

Then,

$$
Z_{\beta_{s}}^{h}\left(g \mid k_{1}, k_{2}\right)_{\alpha_{1}, \ldots, \alpha_{r}}=\int_{\left[\bar{M}_{h}\left(X / \vec{F}, \beta_{s}\right)\right]^{\mathrm{vir}}} \mathrm{ev}_{1}^{*}\left(\alpha_{1}\right) \cup \cdots \cup \mathrm{ev}_{r}^{*}\left(\alpha_{r}\right)
$$

where

$$
\left[\bar{M}_{h}\left(X / \vec{F}, \beta_{s}\right)\right]^{\text {vir }} \in A_{D}^{\mathbb{T}}\left(\bar{M}_{h}\left(X / \vec{F}, \beta_{s}\right)\right)
$$

is in the $D$ th equivariant Chow group for

$$
D=-K_{X} \cdot \beta_{s}=\operatorname{virdim} \bar{M}_{h}\left(X / \vec{F}, \beta_{s}\right) .
$$

Note that the invariants can be non-zero even for negative values of $D$ (c.f. Section 2.1 of [2], and also see Remark 1.5). Then the partition function of the degree $\beta_{s}$, relative, Gromov-Witten invariants is given by

$$
Z_{\beta_{s}}\left(g \mid k_{1}, k_{2}\right)_{\alpha_{1} \cdots \alpha_{r}}=\sum_{h=0}^{\infty} Z_{\beta_{s}}^{h}\left(g \mid k_{1}, k_{2}\right)_{\alpha_{1} \cdots \alpha_{r}} u^{2 h-2-K_{X} \cdot \beta_{s}} .
$$

We can also write the partition function of the section class, relative, Gromov-Witten invariants as

$$
Z\left(g \mid k_{1}, k_{2}\right)_{\alpha_{1} \cdots \alpha_{r}}=\sum_{\beta_{s} \text { is a section class }} Z_{\beta_{s}}\left(g \mid k_{1}, k_{2}\right)_{\alpha_{1} \cdots \alpha_{r}}
$$

${ }^{2}$ We assume that all domain curves are connected (see Remark 2.4). 
It is evident that when $r=0$, we get the partition function for the ordinary invariants defined in Section 1.

Remark 2.1. $Z_{\beta_{s}}\left(g \mid k_{1}, k_{2}\right)_{\alpha_{1} \cdots \alpha_{r}}$ is a homogeneous polynomial in $t_{0}, t_{1}, t_{2}$ of degree

$$
N=\sum_{p=1}^{r} \operatorname{deg}\left(\alpha_{p}\right)-D=\sum_{p=1}^{r} \operatorname{deg}\left(\alpha_{p}\right)+2 g-2-k_{1}-k_{2}-3 n
$$

with coefficients in $\mathbb{Q}((u))$. In particular, it is zero if $N<0$, and it is a Laurent series in $u$, independent of $t_{0}, t_{1}, t_{2}$, when $N=0$ (c.f. [2], Section 2.1).

Remark 2.2. We can reexpress the definition of the partition function for the section class invariants as follows (see Remark 1.4):

$$
Z\left(g \mid k_{1}, k_{2}\right)_{\alpha_{1} \cdots \alpha_{r}}=\sum_{n \in \mathbb{Z}} Z_{\beta_{0}+n f}\left(g \mid k_{1}, k_{2}\right)_{\alpha_{1} \cdots \alpha_{r}}
$$

This sum is finite because by Remark 2.1, it is clear that the sum is terminated from above, and it is also terminated from below because for the large negative values of $n$, there is no curve representing the class $\beta_{0}+n f$, which means that

$$
\bar{M}_{h}\left(X / \vec{F}, \beta_{0}+n f\right)=\emptyset
$$

for $n \ll 0$. To see the last claim, let $E=\mathcal{O} \oplus L_{1} \oplus L_{2}$, and notice that for $n \ll 0$ there is a one to one correspondence between geometric sections representing $\beta_{0}+n f$ (note that for $n \ll 0$, a curve class $\beta_{0}+n f$ must be a geometric section, see Remark 1.2) and degree $-n$ sub-line bundles of $E$. $E$ has no sub-line bundle of degree greater than $k_{1}+k_{2}$. Therefore, for $n \ll 0$, $E$ has no sub-line bundle of degree $-n$, which proves our claim.

Before expressing the gluing theorem, we fix a basis, $\mathcal{B}$, for the equivariant Chow group of $\mathbb{P}^{2}$. We take

$$
\begin{aligned}
& x_{0}:=\left(H-t_{1}\right)\left(H-t_{2}\right), \\
& x_{1}:=\left(H-t_{0}\right)\left(H-t_{1}\right), \\
& x_{2}:=\left(H-t_{0}\right)\left(H-t_{2}\right) .
\end{aligned}
$$


$x_{0}, x_{1}, x_{2}$ are in fact equivariant classes represented by three fixed points of the torus action on $\mathbb{P}^{2}$. We define

$$
\mathcal{B}:=\left\{x_{0}, x_{1}, x_{2}\right\}
$$

It is easy to see that $\mathcal{B}$ is a basis for $A_{*}^{\mathbb{T}}\left(\mathbb{P}^{2}\right) \otimes \mathbb{Q}\left(t_{0}, t_{1}, t_{2}\right)$.

\subsection{Convention}

From now on, we assume that each $\alpha_{p}$ for $p=1, \ldots, r$ in the definition of relative partition functions belongs to this basis set, $\mathcal{B}$.

We take

$$
\begin{aligned}
& T\left(x_{0}\right):=\left(t_{0}-t_{1}\right)\left(t_{0}-t_{2}\right), \\
& T\left(x_{1}\right):=\left(t_{1}-t_{0}\right)\left(t_{1}-t_{2}\right), \\
& T\left(x_{2}\right):=\left(t_{2}-t_{0}\right)\left(t_{2}-t_{1}\right) .
\end{aligned}
$$

We have these relations:

$$
\begin{aligned}
x_{i}^{2} & =T\left(x_{i}\right) x_{i}, \\
x_{i} x_{j} & =0 \quad \text { for } i \neq j .
\end{aligned}
$$

Then we raise the indices for the relative partition functions by the following rule:

$$
Z\left(g \mid k_{1}, k_{2}\right)_{\alpha_{1} \cdots \alpha_{s}}^{\gamma_{1} \cdots \gamma_{t}}:=\left(\prod_{p=1}^{t} \frac{1}{T\left(\gamma_{p}\right)}\right) Z\left(g \mid k_{1}, k_{2}\right)_{\alpha_{1} \cdots \alpha_{s} \gamma_{1} \cdots \gamma_{t}} .
$$

Then we have the following gluing rules similar to Theorem 3.2 in [2]:

Theorem 2.3. For any choices of elements $\alpha_{1}, \ldots, \alpha_{s}$ and $\gamma_{1}, \ldots, \gamma_{t}$ from the set $\mathcal{B}$ and integers satisfying $g=g^{\prime}+g^{\prime \prime}, k_{1}=k_{1}^{\prime}+k_{1}^{\prime \prime}$ and $k_{2}=k_{2}^{\prime}+k_{2}^{\prime \prime}$, we have

$$
Z\left(g \mid k_{1}, k_{2}\right)_{\alpha_{1} \cdots \alpha_{s} \gamma_{1} \cdots \gamma_{t}}=\sum_{\lambda \in \mathcal{B}} Z\left(g^{\prime} \mid k_{1}^{\prime}, k_{2}^{\prime}\right)_{\alpha_{1} \cdots \alpha_{s} \lambda} Z\left(g^{\prime \prime} \mid k_{1}^{\prime \prime}, k_{2}^{\prime \prime}\right)_{\gamma_{1} \cdots \gamma_{t}}^{\lambda},
$$

and

$$
Z\left(g \mid k_{1}, k_{2}\right)_{\alpha_{1} \cdots \alpha_{s}}=\sum_{\lambda \in \mathcal{B}} Z\left(g \mid k_{1}, k_{2}\right)_{\alpha_{1} \cdots \alpha_{s} \lambda}^{\lambda} .
$$


The proof of this theorem will be given in Appendix A.

Remark 2.4. In most of the contexts in which the relative Gromov-Witten invariants are being used, maps with disconnected domain curves are considered as well as ones with connected domains. In Lemma A.1, we prove that in our case, where we only deal with section classes, we do not need to consider disconnected domain curves.

Remark 2.5. In exactly the same way as in [2], one can prove by using Theorem 2.3 that the partition functions $Z(g \mid 0,0)_{\alpha_{1} \cdots \alpha_{r}}$ give rise to a $1+1$-dimensional TQFT taking values in the $\operatorname{ring} R=\mathbb{Q}((u))\left(t_{0}, t_{1}, t_{2}\right)$. The Frobenius algebra corresponding to this TQFT (see [1], Theorem 2.1) is

$$
H=\bigoplus_{i=0}^{2} \operatorname{Re}_{x_{i}}
$$

for $x_{i} \in \mathcal{B}$, with multiplication given by

$$
e_{x_{i}} \otimes e_{x_{j}}=\sum_{k=0}^{2} Z(g \mid 0,0)_{x_{i} x_{j}}^{x_{k}} e_{k} .
$$

We will prove that this Frobenius algebra and hence the corresponding TQFT is semi-simple (Proposition 3.11). In Section 5, we use this fact for proving Theorem 1.6 and 1.7 for the case $g=0$.

We will use the following corollary of Theorem 2.3 in our calculations:

Corollary 2.6. With the same notation as in Theorem 2.3, we have

$$
\begin{aligned}
& Z_{\beta_{0}+n f}\left(g \mid k_{1}, k_{2}\right)_{\alpha_{1} \cdots \alpha_{s} \gamma_{1} \cdots \gamma_{t}} \\
& \quad=\sum_{\lambda \in \mathcal{B}} \sum_{n=n^{\prime}+n^{\prime \prime}} Z_{\beta_{0}+n^{\prime} f}\left(g^{\prime} \mid k_{1}^{\prime}, k_{2}^{\prime}\right)_{\alpha_{1} \cdots \alpha_{s} \lambda} Z_{\beta_{0}+n^{\prime \prime} f}\left(g^{\prime \prime} \mid k_{1}^{\prime \prime}, k_{2}^{\prime \prime}\right)_{\gamma_{1} \ldots \gamma_{t}}^{\lambda}
\end{aligned}
$$

\section{Calculations}

We will work with the space

$$
X=\mathbb{P}\left(\mathcal{O} \oplus L_{1} \oplus L_{2}\right) \rightarrow\left(C, p_{1}, \ldots, p_{r}\right)
$$

throughout this section. In accordance with the notations in [2], we will use the words cap, tube and pants to refer to the case where the base curve, $C$, 
is a genus zero curve with one, two and three marked points, respectively (see Remark 1.5). We sometimes refer to the partition functions by referring to the space to which they correspond. We will use the notation

$$
\phi:=2 \sin \frac{u}{2}
$$

in the later calculations.

Similar to Section 4.3 in [2], one can see that the following partition functions determine the theory completely:

$$
\begin{aligned}
& Z(0 \mid 0,0)_{\alpha}, \quad Z(0 \mid 0,0)_{\alpha_{1} \alpha_{2}}, \quad Z(0 \mid 0,0)_{\alpha_{1} \alpha_{2} \alpha_{3}}, \quad Z(0 \mid-1,0)_{\alpha}, \\
& Z(0 \mid 0,-1)_{\alpha}, \quad Z(0 \mid 1,0)_{\alpha}, \quad Z(0 \mid 0,1)_{\alpha} .
\end{aligned}
$$

We refer to the partition functions above as the basic partition functions.

By the discussion given in Remark 2.2, one can prove the following lemma:

Lemma 3.1. The basic partition functions are given by

$$
\begin{aligned}
& Z(0 \mid 0,0)_{\alpha}=Z_{\beta_{0}}(0 \mid 0,0)_{\alpha}, \quad Z(0 \mid 0,0)_{\alpha_{1} \alpha_{2}}=Z_{\beta_{0}}(0 \mid 0,0)_{\alpha_{1} \alpha_{2}}, \\
& Z(0 \mid-1,0)_{\alpha}=Z_{\beta_{0}}(0 \mid-1,0)_{\alpha}, \quad Z(0 \mid 0,-1)_{\alpha}=Z_{\beta_{0}}(0 \mid 0,-1)_{\alpha}, \\
& Z(0 \mid 1,0)_{\alpha}=Z_{\beta_{0}-f}(0 \mid 1,0)_{\alpha}, \quad Z(0 \mid 0,1)_{\alpha}=Z_{\beta_{0}-f}(0 \mid 0,1)_{\alpha}, \\
& Z(0 \mid 0,0)_{\alpha_{1} \alpha_{2} \alpha_{3}}=Z_{\beta_{0}}(0 \mid 0,0)_{\alpha_{1} \alpha_{2} \alpha_{3}}+Z_{\beta_{0}+f}(0 \mid 0,0)_{\alpha_{1} \alpha_{2} \alpha_{3}} .
\end{aligned}
$$

Proof. We prove the last equality as follows. In the right hand side, we do not have any partition function of degree $\beta_{0}+n f$ for $n<0$, because $\mathcal{O} \oplus L_{1} \oplus L_{2}$ does not have any sub-line bundle of a positive degree, as $L_{1}$ and $L_{2}$ are of degree zero. We also do not have any partition function of degree $\beta_{0}+n f$ for $n>1$ because

$$
\begin{aligned}
N & =\sum_{p=1}^{3} \operatorname{deg}\left(\alpha_{p}\right)-D \\
& =(2+2+2)-(3 H+2 F) \cdot\left(\beta_{0}+n f\right)=4-3 n
\end{aligned}
$$

which is negative for $n>1$ (see Remark 2.1). The other equalities are proved similarly.

The rest of this section is devoted to computing the terms that appeared in the right hand sides of the equations in Lemma 3.1. 


\subsection{Calculations via localization}

The complex torus acts on $X$ as before. We define

$S_{0}$ : the locus of $(1: 0: 0) \quad$ in $X \cong \mathbb{P}\left(\mathcal{O} \oplus L_{1} L_{0}^{-1} \oplus L_{2} L_{0}^{-1}\right)$,

$S_{1}$ : the locus of $(0: 1: 0)$ in $X \cong \mathbb{P}\left(L_{0} L_{1}^{-1} \oplus \mathcal{O} \oplus L_{2} L_{1}^{-1}\right)$,

$S_{2}$ : the locus of $(0: 0: 1)$ in $X \cong \mathbb{P}\left(L_{0} L_{2}^{-1} \oplus L_{1} L_{2}^{-1} \oplus \mathcal{O}\right)$.

It is clear that $S_{0}, S_{1}$ and $S_{2}$ are fixed under the torus action on $X$. One can also see that $S_{0}, S_{1}$ and $S_{2}$ represent the classes $\beta_{0}, \beta_{0}-k_{1} f$ and $\beta_{0}-k_{2} f$, respectively.

As before, let $\beta_{s}$ be a section class. The torus action on $X$ induces an action on $\bar{M}_{h}\left(X / \vec{F}, \beta_{s}\right)$. We denote the fixed locus of this action by $\bar{M}_{h}\left(X / \vec{F}, \beta_{s}\right)^{\mathbb{T}}$.

By notations of Section 2 , we let $S_{i}[\vec{L}] \subset X[\vec{L}]$ be the $l_{p}$-step degeneration of $S_{i}$ along intersection points $\tau_{i p}=S_{i} \cap F_{p}$ for $p=1, \ldots, r$, and $i=0,1,2$, such that $S_{i}[\vec{L}]$ is still fixed under the induced actions on $X[\vec{L}]$. Then, $\bar{M}_{h}\left(X / \vec{F}, \beta_{s}\right)^{\mathbb{T}}$ parameterizes maps $q: C^{\prime} \rightarrow X[\vec{L}]$ for some $\vec{L}$, whose images are either of $S_{i}[\vec{L}] \cup_{n=1}^{m_{i}} f_{n}$ for $i=0,1$ or 2 , where by the last expression we mean $S_{i}[\vec{L}]$ with $m_{i} \mathbb{T}$-fixed fiber curves, $f_{n}\left(f_{n}\right.$ represents the class $b f$ for some $\left.b \in \mathbb{Z}^{+}\right)$, are attached to it at some points. Note that the choice of $i \in\{0,1,2\}$, and also the number of fibers which are attached to $S_{i}[\vec{L}], m_{i}$, are constrained by the class $\beta_{s}$.

In general, the moduli space $\bar{M}_{h}\left(X / \vec{F}, \beta_{s}\right)^{\mathbb{T}}$ can be quite complicated because of the existence of the fibers attached to each $S_{i}[\vec{L}]$. However, in the special case where $m_{i}=0$ for some $i$ (i.e., there is no fiber attached to $\left.S_{i}[\vec{L}]\right)$, it is evident that the corresponding component of $\bar{M}_{h}\left(X / \vec{F}, \beta_{s}\right)^{\mathbb{T}}$ (parameterizing maps with images equal to $S_{i}[\vec{L}]$ ) is the moduli space of degree one relative stable maps to curves, which we denote by $\bar{M}_{h}\left(S_{i} / \vec{\tau}_{i}, 1\right)$, where $\vec{\tau}_{i}=\left(\tau_{i 1}, \ldots, \tau_{i r}\right)$.

\subsection{Assumption}

For the rest of Section 3.1, we assume that

$$
\bar{M}_{h}\left(X / \vec{F}, \beta_{s}\right)^{\mathbb{T}}=\bigcup_{i \in I} \bar{M}_{h}\left(S_{i} / \vec{\tau}_{i}, 1\right)
$$

where $I \subset\{0,1,2\}$, depending on the class $\beta_{s}$. 
Then one can see that the $\mathbb{T}$-fixed part of the perfect obstruction theory of $\bar{M}_{h}\left(X / \vec{F}, \beta_{s}\right)$ is exactly the usual obstruction theory of $\bigcup_{i \in I} \bar{M}_{h}\left(S_{i} / \vec{\tau}_{i}, 1\right)$, and therefore

$$
\left[\bar{M}_{h}\left(X / \vec{F}, \beta_{s}\right)^{\mathbb{T}}\right]^{\mathrm{vir}} \cong \sum_{i \in I}\left[\bar{M}_{h}\left(S_{i} / \vec{\tau}_{i}, 1\right)\right]^{\mathrm{vir}}
$$

In the special case where $m_{i}=0$ for all possible $i$, the assumption above holds. One can see easily that this is the case for all the partition functions in the right hand sides of equations in Lemma 3.1, except for $Z_{\beta_{0}+f}(0 \mid 0,0)_{\alpha_{1} \alpha_{2} \alpha_{3}}$.

In this section, we will use localization for calculating the former partition functions. $Z_{\beta_{0}+f}(0 \mid 0,0)_{\alpha_{1} \alpha_{2} \alpha_{3}}$ will be calculated in Section 3.3 by combining the results of this section with the gluing techniques.

Applying the relative virtual localization formula (see Section 3 of $[5]^{3}$ ), we can write

$$
\begin{aligned}
& Z_{\beta_{s}}\left(g \mid k_{1}, k_{2}\right)_{\alpha_{1} \cdots \alpha_{r}} \\
& \quad=\sum_{i \in I} \sum_{h=0}^{\infty} u^{2 h-2-K_{X} \cdot \beta_{s}} \int_{\left[\bar{M}_{h}\left(S_{i} / \vec{\tau}_{i}, 1\right)\right]^{\mathrm{vir}}} \frac{\mathrm{ev}_{1}^{*}\left(\alpha_{1}\right) \cap \cdots \cap \mathrm{ev}_{r}^{*}\left(\alpha_{r}\right)}{e\left(\operatorname{Norm}_{i}^{\text {vir }}\right)}
\end{aligned}
$$

where Norm $_{i}^{\text {vir }}$ is the equivariant virtual normal bundle of the component of the $\mathbb{T}$-fixed loci in $\bar{M}_{h}\left(X / \vec{F}, \beta_{s}\right)$, and $e\left(\right.$ Norm $\left._{i}^{\text {vir }}\right)$ is its equivariant Euler class.

Let $\pi: U \rightarrow \bar{M}_{h}\left(X / \vec{F}, \beta_{s}\right)$ and $q: U \rightarrow X$ be the universal curve and the universal map, respectively, and let $N_{S_{i} / X}$ be the normal bundle of $S_{i}$ in $X$. Then we have the following lemma for evaluating $e\left(\operatorname{Norm}_{i}^{\text {vir }}\right)$ :

Lemma 3.2. For each $i \in I$, the equivariant Euler class of the virtual normal bundle of the component of the fixed loci, which is isomorphic to $\bar{M}_{h}\left(S_{i} / \vec{\tau}_{i}, 1\right)$, is given by

$$
e\left(\operatorname{Norm}_{i}^{\mathrm{vir}}\right)=e\left(R^{\bullet} \pi_{*} q^{*} N_{S_{i}} / X\right)=e\left(R^{\bullet} \pi_{*} q^{*}\left(L_{j} L_{i}^{-1} \oplus L_{k} L_{i}^{-1}\right)\right),
$$

where $j$ and $k$ are two distinct elements of $\{1,2,3\}-\{i\}$.

Proof. Following the notations of [5], for a normal crossings divisor $D$ contained in the smooth locus of a variety $V$, by $T_{V}(-\log D)$, we mean the dual

\footnotetext{
${ }^{3}$ In [5], the authors assume for convenience that the relative divisor is in the fixed locus, but it is straight forward to adapt their methods to the case at hand [4].
} 
of the sheaf of Kähler differentials with logarithmic poles along $D$. Let

$$
\tilde{q}: C^{\prime} \longrightarrow S_{i}[\vec{L}] \longleftrightarrow X[\vec{L}]
$$

be a relative map corresponding to an element of $\bar{M}_{h}\left(S_{i} / \vec{\tau}_{i}, 1\right)$ and

$$
\mathrm{c}: X[\vec{L}] \rightarrow X
$$

be the natural contraction map. For the divisor $D \subset V$ as above, and a given integer $l \geq 0$, let $D_{\infty}$ be the infinity section of the $l$ th component of the $l$-step degeneration of $V$ along $D$ (see Section 2.1 of [5] for more details). Note that when $l=0, D_{\infty}$ is $D$. Then by the description of $T_{V}(-\log D)$ given in Section 2.8 of [5], we have the following equality in $K$-theory

$$
\rho_{i}^{*} T_{X[L]}\left(-\sum_{p=1}^{r} \log F_{p, \infty}\right)=T_{S_{i}[L]}\left(-\sum_{p=1}^{r} \log \tau_{i p, \infty}\right) \oplus \mathrm{c}^{*} N_{S_{i} / X}
$$

where $\rho_{i}$ is the inclusion of $S_{i}[\vec{L}]$ into $X[\vec{L}]$, and hence

$$
\begin{aligned}
& H^{*}\left(C^{\prime}, \tilde{q}^{*} T_{X[L]}\left(-\sum_{p=1}^{r} \log F_{p, \infty}\right)\right) \\
& =H^{*}\left(C^{\prime}, \tilde{q}^{*} T_{S_{i}[L]}\left(-\sum_{p=1}^{r} \log \tau_{i p, \infty}\right)\right) \oplus H^{*}\left(C^{\prime}, \tilde{q}^{*} c^{*} N_{S_{i} / X}\right) .
\end{aligned}
$$

The first equality in the lemma follows from this, and a comparison of the moving parts of the obstruction/deformation sequences of $\bar{M}_{h}\left(X / \vec{F}, \beta_{s}\right)$ and $\bar{M}_{h}\left(S_{i} / \vec{\tau}_{i}, 1\right)$ under the induced torus action (See (2) and (3) in [5]), and noting that we consider degree 1 maps to $S_{i}[\vec{L}]$.

The second equality in the lemma follows from the isomorphism:

$$
N_{S_{i} / X} \cong L_{j} L_{i}^{-1} \oplus L_{k} L_{i}^{-1}
$$

Remark 3.3. The last expression in Lemma 3.2 is consistent with the notations of Section 2.2 of [2], where the authors considered the moduli space of maps from curves to the total space of a direct sum of two line bundles over a curve. 
For manipulating the evaluation functions in (3.2), we use the following Cartesian diagram for each $p=1, \ldots, r$, and $i \in I$ :

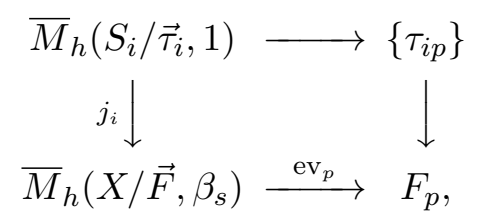

where two vertical maps are inclusions, and $\tau_{i p}$ is the intersection point of $S_{i}$ with $F_{p}$, which is the fixed point of the torus action on $F_{p}$ representing the class $x_{i} \in \mathcal{B}$. From this diagram, it is clear that $\operatorname{ev}_{p}^{*}\left(\alpha_{p}\right)$, restricted to $\bar{M}_{h}\left(S_{i} / \vec{\tau}_{i}, 1\right)$, is a class of pure weight for each $p$ and can be taken out of the integrals.

We summarize all the discussion above in the following equation:

$$
\begin{aligned}
Z_{\beta}\left(g \mid k_{1}, k_{2}\right)_{\alpha_{1} \cdots \alpha_{r}}= & \sum_{h=0}^{\infty} u^{2 h-2-K_{X} \cdot \beta_{s}} \sum_{i \in I}\left(\prod_{p=1}^{r}\left(\operatorname{ev}_{p} \circ j_{i}\right)^{*}\left(\alpha_{p}\right)\right) \\
& \times \int_{\left[\bar{M}_{h}\left(S_{i} / \vec{\tau}_{i}, 1\right)\right]^{\mathrm{vir}}} e\left(-R^{\bullet} \pi_{*} q^{*}\left(N_{S_{i} / X}\right)\right) .
\end{aligned}
$$

By chasing the diagram above, one can see easily that

$$
\left(\operatorname{ev}_{p} \circ j_{i}\right)^{*}\left(x_{k}\right)= \begin{cases}T\left(x_{i}\right) & \text { if } i=k \\ 0 & \text { otherwise }\end{cases}
$$

for $k \in\{0,1,2\}$ (see Section 2 for the definition of $T(-)$ ).

\subsubsection{Computing degree $\beta_{0}$, level $(0,0)$ cap, tube and pants}

Lemma 3.4. Partition functions for the degree $\beta_{0}$, level $(0,0)$ cap, tube and pants are given by

$$
\begin{aligned}
Z_{\beta_{0}}(0 \mid 0,0)_{x_{a}} & =1 \\
Z_{\beta_{0}}(0 \mid 0,0)_{x_{a} x_{b}} & = \begin{cases}T\left(x_{a}\right) & \text { if } a=b \\
0 & \text { otherwise }\end{cases} \\
Z_{\beta_{0}}(0 \mid 0,0)_{x_{a} x_{b} x_{c}} & = \begin{cases}T\left(x_{a}\right)^{2} & \text { if } a=b=c \\
0 & \text { otherwise }\end{cases}
\end{aligned}
$$

for $a, b, c \in\{0,1,2\}$. 
Proof. Since $k_{1}=k_{2}=0$, all $S_{0}, S_{1}$ and $S_{2}$ represent the class $\beta_{0}$, and so in (3.3) we have $I=\{0,1,2\}$.

We use the results of Sections 6.2 and 6.4.2 in [2] to evaluate the integrals in (3.3), for the cap, tube and pants. We prove the formula for the tube, and the other cases are similar. By Lemma 6.1 in [2] (for $d=1$ ), and Lemma 3.2, and also by (3.3) and (3.4), we have

$$
\begin{aligned}
Z_{\beta_{0}}(0 \mid 0,0)_{x_{a} x_{b}}= & \sum_{h=0}^{\infty} u^{2 h-2-K_{X} \cdot \beta_{0}}\left(\left(\mathrm{ev}_{1} \circ j_{0}\right)^{*}\left(x_{a}\right)\left(\mathrm{ev}_{2} \circ j_{0}\right)^{*}\left(x_{b}\right)\right. \\
& \cdot \int_{\left[\bar{M}_{h}\left(S_{0} /\left(\tau_{01}, \tau_{02}\right), 1\right)\right]^{\mathrm{vir}}} e\left(-R^{\bullet} \pi_{*} q^{*}\left(L_{1} L_{0}^{-1} \oplus L_{2} L_{0}^{-1}\right)\right) \\
& +\left(\mathrm{ev}_{1} \circ j_{1}\right)^{*}\left(x_{a}\right)\left(\mathrm{ev}_{2} \circ j_{1}\right)^{*}\left(x_{b}\right) \\
& \cdot \int_{\left[\bar{M}_{h}\left(S_{1} /\left(\tau_{11}, \tau_{12}\right), 1\right)\right]^{\mathrm{vir}}} e\left(-R^{\bullet} \pi_{*} q^{*}\left(L_{0} L_{1}^{-1} \oplus L_{2} L_{1}^{-1}\right)\right) \\
& +\left(\mathrm{ev}_{1} \circ j_{2}\right)^{*}\left(x_{a}\right)\left(\mathrm{ev}_{2} \circ j_{2}\right)^{*}\left(x_{b}\right) \\
& \left.\cdot \int_{\left[\bar{M}_{h}\left(S_{2} /\left(\tau_{21}, \tau_{22}\right), 1\right)\right]^{\mathrm{vir}}} e\left(-R^{\bullet} \pi_{*} q^{*}\left(L_{0} L_{2}^{-1} \oplus L_{1} L_{2}^{-1}\right)\right)\right) \\
= & \sum_{i=0}^{2}\left(\delta_{a}^{i} T\left(x_{i}\right)\right)\left(\delta_{b}^{i} T\left(x_{i}\right)\right) \frac{1}{T\left(x_{i}\right)} \\
= & \delta_{a}^{b} T\left(x_{a}\right) .
\end{aligned}
$$

Note that the weights of the torus action on the first and the second factors of $L_{j} L_{i}^{-1} \oplus L_{k} L_{i}^{-1}$ are $t_{i}-t_{j}$ and $t_{i}-t_{k}$, respectively (which they play the roles of $t_{1}$ and $t_{2}$ in Lemma 6.1 in [2]).

3.2.2. Computing degree $\beta_{0}$, level $(0,-1)$ and $(-1,0)$ and degree $\beta_{0}-f$, level $(0,1)$ and $(1,0)$ caps

Lemma 3.5. Partition functions for the degree $\beta_{0}$, level $(0,-1)$ and $(-1,0)$ caps are given by

$$
\begin{aligned}
& Z_{\beta_{0}}(0 \mid 0,-1)_{x_{a}}=\left(t_{a}-t_{2}\right) \phi^{-1} \\
& Z_{\beta_{0}}(0 \mid-1,0)_{x_{a}}=\left(t_{a}-t_{1}\right) \phi^{-1}
\end{aligned}
$$

for $a=0,1,2$.

Proof. We prove the first formula, and the second one is proved in a similar way. We have $k_{1}=0$ and $k_{2}=-1, S_{0}, S_{1}$ represent the class $\beta_{0}$, but $S_{2}$ represents the class $\beta_{0}+f$. Therefore in (3.3) we have $I=\{0,1\}$. 
By Lemma 6.3 in [2] (for $d=1$ ), and also Lemma 3.2 and (3.4), we can rewrite (3.3) as

$$
\begin{aligned}
Z_{\beta_{0}}(0 \mid 0,-1)_{x_{a}}= & \sum_{h=0}^{\infty} u^{2 h-2-K_{X} \cdot \beta_{0}}\left(\left(\mathrm{ev}_{1} \circ j_{0}\right)^{*}\left(x_{a}\right) \int_{\left[\bar{M}_{h}\left(S_{0} / \tau_{01}, 1\right)\right]^{\mathrm{vir}}}\right. \\
& \times e\left(-R^{\bullet} \pi_{*} q^{*}\left(L_{1} L_{0}^{-1} \oplus L_{2} L_{0}^{-1}\right)\right) \\
& +\left(\mathrm{ev}_{1} \circ j_{1}\right)^{*}\left(x_{a}\right) \int_{\left[\bar{M}_{h}\left(S_{1} / \tau_{11}, 1\right)\right] \mathrm{vir}} \\
& \left.\times e\left(-R^{\bullet} \pi_{*} q^{*}\left(L_{0} L_{1}^{-1} \oplus L_{2} L_{1}^{-1}\right)\right)\right) \\
= & \left(\frac{\delta_{a}^{0} T\left(x_{0}\right)}{t_{0}-t_{1}}+\frac{\delta_{a}^{1} T\left(x_{1}\right)}{t_{1}-t_{0}}\right) \phi^{-1} \\
= & \left(t_{a}-t_{2}\right) \phi^{-1} .
\end{aligned}
$$

Lemma 3.6. Partition functions for the degree $\beta_{0}-f$, level $(0,1)$ and $(1,0)$ caps are given by

$$
\begin{aligned}
& Z_{\beta_{0}-f}(0 \mid 0,1)_{x_{a}}=\left(t_{a}-t_{0}\right)\left(t_{a}-t_{1}\right) \phi^{-2} \\
& Z_{\beta_{0}-f}(0 \mid 1,0)_{x_{a}}=\left(t_{a}-t_{0}\right)\left(t_{a}-t_{2}\right) \phi^{-2}
\end{aligned}
$$

for $a=0,1,2$.

Proof. For the first relation, note that only $S_{2}$ represents the class $\beta_{0}-f$, and so we have $I=\{2\}$. Note also that the normal bundle of $S_{2}$ in $X$ is level $(-1,-1)$ in this case. The rest of the proof is quite similar to the proof of Lemma 3.5, except that this time the relevant integral is obtained by applying Theorem 3.2 in [2] to the level $(0,-1)$ and $(-1,0)$ caps (given by Lemma 6.3 in [2]) and the level $(0,0)$ pants (given in Section 6.4.2 in [2]) to get the level $(-1,-1)$ cap.

\subsection{Calculations via gluing techniques}

In this section, we use Corollary 2.6 (which is referred to as the gluing formula), and the results of Section 3.1 to find $Z_{\beta_{0}+f}(0 \mid 0,0)_{\alpha_{1} \alpha_{2} \alpha_{3}}$. For a treatment of gluing spaces and applying the gluing theorem, see Appendix A. 
We first need to find the following partition functions of tubes:

$$
\begin{aligned}
Z(0 \mid 0,-1)_{\alpha_{1} \alpha_{2}} & =Z_{\beta_{0}}(0 \mid 0,-1)_{\alpha_{1} \alpha_{2}}+Z_{\beta_{0}+f}(0 \mid 0,-1)_{\alpha_{1} \alpha_{2}}, \\
Z(0 \mid 0,1)_{\alpha_{1} \alpha_{2}} & =Z_{\beta_{0}-f}(0 \mid 0,1)_{\alpha_{1} \alpha_{2}}+Z_{\beta_{0}}(0 \mid 0,1)_{\alpha_{1} \alpha_{2}} .
\end{aligned}
$$

Similar relations hold after swapping the degrees. These equalities can be proven similar to the proof of Lemma 3.1.

3.3.1. Computing degree $\beta_{0}$, level $(0,-1)$ and $(-1,0)$ and degree $\beta_{0}-f$, level $(0,1)$ and $(1,0)$ tubes

Lemma 3.7. Partition functions for the degree $\beta_{0}$, level $(0,-1)$ and $(-1,0)$ tubes are given by

$$
\begin{aligned}
& Z_{\beta_{0}}(0 \mid 0,-1)_{x_{a} x_{b}}= \begin{cases}\left(t_{0}-t_{1}\right)\left(t_{0}-t_{2}\right)^{2} \phi^{-1} & \text { if } a=b=0, \\
\left(t_{1}-t_{0}\right)\left(t_{1}-t_{2}\right)^{2} \phi^{-1} & \text { if } a=b=1, \\
0 & \text { otherwise. }\end{cases} \\
& Z_{\beta_{0}}(0 \mid-1,0)_{x_{a} x_{b}}= \begin{cases}\left(t_{0}-t_{2}\right)\left(t_{0}-t_{1}\right)^{2} \phi^{-1} & \text { if } a=b=0, \\
\left(t_{2}-t_{0}\right)\left(t_{2}-t_{1}\right)^{2} \phi^{-1} & \text { if } a=b=2, \\
0 & \text { otherwise }\end{cases}
\end{aligned}
$$

for $a, b \in\{0,1,2\}$.

Proof. The first relation is simply proved by attaching the level $(0,-1)$ cap to the level $(0,0)$ pants and applying the gluing formula. This is schematically indicated by the following picture:

$$
\begin{aligned}
& x_{a}\left(\sum_{\beta_{0}}^{(0,-1)}\right) x_{a}=\overbrace{\beta_{0}}^{(0,-1) x_{a}^{(0,0)}} \sum_{\beta_{0}}^{()_{a}} x_{a} \\
& Z_{\beta_{0}}(0 \mid 0,-1)_{x_{a} x_{a}}=Z_{\beta_{0}}(0 \mid 0,-1)_{x_{a}} Z_{\beta_{0}}(0 \mid 0,0)_{x_{a} x_{a}}^{x_{a}} .
\end{aligned}
$$

The result is now obvious by applying Lemmas 3.5 and 3.6. The proof of the second relation is similar. 
Similar to the proof of Lemma 3.7, we can prove this:

Lemma 3.8. Partition functions for the degree $\beta_{0}-f$, level $(0,1)$ and $(1,0)$ tubes are given by

$$
\begin{aligned}
& Z_{\beta_{0}-f}(0 \mid 0,1)_{x_{a} x_{b}}= \begin{cases}\left(t_{2}-t_{0}\right)^{2}\left(t_{2}-t_{1}\right)^{2} \phi^{-2} & \text { if } a=b=2, \\
0 & \text { otherwise. }\end{cases} \\
& Z_{\beta_{0}-f}(0 \mid 1,0)_{x_{a} x_{b}}= \begin{cases}\left(t_{1}-t_{2}\right)^{2}\left(t_{1}-t_{0}\right)^{2} \phi^{-2} & \text { if } a=b=1, \\
0 & \text { otherwise }\end{cases}
\end{aligned}
$$

for $a, b \in\{0,1,2\}$.

3.3.2. Computing degree $\boldsymbol{\beta}_{0}$, level $(0,1)$ and $(1,0)$, and also degree $\boldsymbol{\beta}_{\mathbf{0}}+\boldsymbol{f}$, level $(\mathbf{0}, \mathbf{- 1})$ and $(\mathbf{- 1 , 0 )}$ tubes We do the calculations for degree $\beta_{0}$, level $(0,1)$ and degree $\beta_{0}+f$, level $(0,-1)$ tubes, the $(1,0)$ and $(-1,0)$ cases are similar.

We attach two tubes of levels $(0,-1)$ and $(0,1)$ to get a tube of level $(0,0)$ (see the picture). Now applying gluing formula and using Lemmas 3.7 and 3.8 , we get

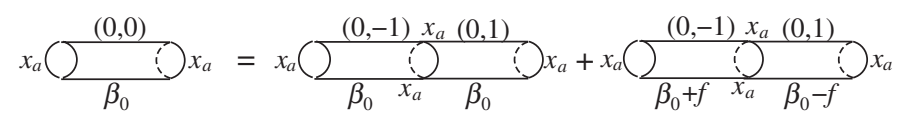

$$
\begin{aligned}
& Z_{\beta_{0}}(0 \mid 0,0)_{x_{a} x_{a}}=Z_{\beta_{0}}(0 \mid 0,-1)_{x_{a} x_{a}} Z_{\beta_{0}}(0 \mid 0,1)_{x_{a}}^{x_{a}} \\
& +Z_{\beta_{0}+f}(0 \mid 0,-1)_{x_{a} x_{a}} Z_{\beta_{0}-f}(0 \mid 0,1)_{x_{a}}^{x_{a}}
\end{aligned}
$$

for $a=0,1,2$.

By using Lemmas 3.4, 3.7 and 3.8, we can solve the equations above for the other unknowns:

$$
\begin{aligned}
Z_{\beta_{0}}(0 \mid 0,1)_{x_{0} x_{0}} & =\left(t_{0}-t_{1}\right) \phi \\
Z_{\beta_{0}}(0 \mid 0,1)_{x_{1} x_{1}} & =\left(t_{1}-t_{0}\right) \phi \\
Z_{\beta_{0}+f}(0 \mid 0,-1)_{x_{2} x_{2}} & =\phi^{2}
\end{aligned}
$$

By changing relative conditions, we can get more relations:

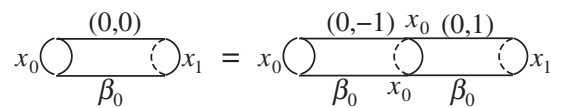

$$
\begin{aligned}
& 0=Z_{\beta_{0}}(0 \mid 0,0)_{x_{0} x_{1}}=Z_{\beta_{0}}(0 \mid 0,-1)_{x_{0} x_{0}} Z_{\beta_{0}}(0 \mid 0,1)_{x_{1}}^{x_{0}} \text {, }
\end{aligned}
$$


which implies that

$$
Z_{\beta_{0}}(0 \mid 0,1)_{x_{0} x_{1}}=0
$$

We can also write (by using Lemmas 3.7 and 3.8):

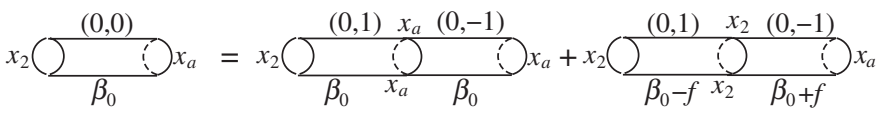

$$
\begin{aligned}
& 0=Z_{\beta_{0}}(0 \mid 0,0)_{x_{2} x_{a}}=Z_{\beta_{0}}(0 \mid 0,1)_{x_{2} x_{a}} Z_{\beta_{0}}(0 \mid 0,-1)_{x_{a}}^{x_{a}} \\
& +Z_{\beta_{0}-f}(0 \mid 0,1)_{x_{2} x_{2}} Z_{\beta_{0}+f}(0 \mid 0,-1)_{x_{a}}^{x_{2}}
\end{aligned}
$$

for $a=0,1$. This with Lemmas 3.7 and 3.8 implies that

$$
\begin{aligned}
& Z_{\beta_{0}}(0 \mid 0,1)_{x_{0} x_{2}}=Z_{\beta_{0}+f}(0 \mid 0,-1)_{x_{0} x_{2}}\left(t_{2}-t_{1}\right) \phi^{-1} \\
& Z_{\beta_{0}}(0 \mid 0,1)_{x_{1} x_{2}}=Z_{\beta_{0}+f}(0 \mid 0,-1)_{x_{1} x_{2}}\left(t_{2}-t_{0}\right) \phi^{-1} .
\end{aligned}
$$

Attaching the level $(0,0)$ cap to the level $(0,1)$ tube, we get three relations:

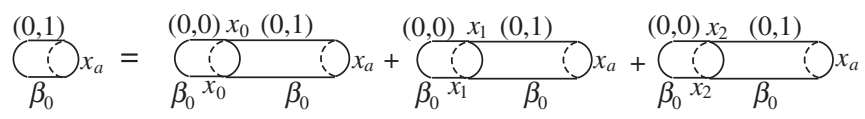

$$
\begin{aligned}
& 0=Z_{\beta_{0}}(0 \mid 0,1)_{x_{a}}^{x_{0}}+Z_{\beta_{0}}(0 \mid 0,1)_{x_{a}}^{x_{1}}+Z_{\beta_{0}}(0 \mid 0,1)_{x_{a}}^{x_{2}}
\end{aligned}
$$

for $a=0,1,2$. We already know that

$$
Z_{\beta_{0}}(0 \mid 0,1)_{x_{0}}^{x_{1}}=Z_{\beta_{0}}(0 \mid 0,1)_{x_{1}}^{x_{0}}=0,
$$

so we get

$$
\begin{aligned}
Z_{\beta_{0}}(0 \mid 0,1)_{x_{0} x_{2}} & =\left(t_{2}-t_{1}\right) \phi \\
Z_{\beta_{0}}(0 \mid 0,1)_{x_{1} x_{2}} & =\left(t_{2}-t_{0}\right) \phi \\
Z_{\beta_{0}}(0 \mid 0,1)_{x_{2} x_{2}} & =\left(2 t_{2}-t_{0}-t_{1}\right) \phi .
\end{aligned}
$$

Combining with (3.6), we find

$$
Z_{\beta_{0}+f}(0 \mid 0,-1)_{x_{0} x_{2}}=Z_{\beta_{0}+f}(0 \mid 0,-1)_{x_{1} x_{2}}=\phi^{2} .
$$


By writing more relations in a similar way (see the pictures, $a=0,1$ ), we can prove

$$
\begin{aligned}
& Z_{\beta_{0}+f}(0 \mid 0,-1)_{x_{0} x_{0}}=Z_{\beta_{0}+f}(0 \mid 0,-1)_{x_{1} x_{1}}=Z_{\beta_{0}+f}(0 \mid 0,-1)_{x_{0} x_{1}}=\phi^{2} .
\end{aligned}
$$

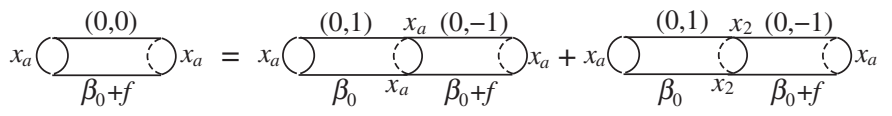

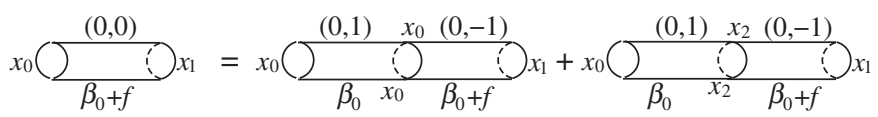

We now summarize all we have proven in this section into the following lemma:

Lemma 3.9. Partition functions for the degree $\beta_{0}$, level $(0,1)$ and $(1,0)$ tubes and also for the degree $\beta_{0}+f$, level $(0,-1)$ and $(-1,0)$ tubes are given by

$$
\begin{aligned}
{\left[Z_{\beta_{0}}(0 \mid 0,1)_{x_{a} x_{b}}\right]=} & {\left[\begin{array}{ccc}
t_{0}-t_{1} & 0 & t_{2}-t_{1} \\
0 & t_{1}-t_{0} & t_{2}-t_{0} \\
t_{2}-t_{1} & t_{2}-t_{0} & 2 t_{2}-t_{0}-t_{1}
\end{array}\right] \phi, } \\
{\left[Z_{\beta_{0}}(0 \mid 1,0)_{x_{a} x_{b}}\right]=} & {\left[\begin{array}{ccc}
t_{0}-t_{2} & t_{1}-t_{2} & 0 \\
t_{1}-t_{2} & 2 t_{1}-t_{0}-t_{2} & t_{1}-t_{0} \\
0 & t_{1}-t_{0} & t_{2}-t_{0}
\end{array}\right] \phi, } \\
{\left[Z_{\beta_{0}+f}(0 \mid 0,-1)_{x_{a} x_{b}}\right]=} & {\left[\begin{array}{lll}
1 & 1 & 1 \\
1 & 1 & 1 \\
1 & 1 & 1
\end{array}\right] \phi^{2}, } \\
{\left[Z_{\beta_{0}+f}(0 \mid-1,0)_{x_{a} x_{b}}\right]=} & {\left[\begin{array}{lll}
1 & 1 & 1 \\
1 & 1 & 1 \\
1 & 1 & 1
\end{array}\right] \phi^{2}, }
\end{aligned}
$$

for $a, b \in\{0,1,2\}$, where partition functions with the index $x_{a} x_{b}$ are the $(a+1, b+1)$ entry of the matrices above. 


\subsubsection{Computing degree $\beta_{0}+f$, level $(0,0)$ pants}

Lemma 3.10. Partition functions for the degree $\beta_{0}+f$, level $(0,0)$ pants are given by

$$
\begin{array}{lc}
Z_{\beta_{0}+f}(0 \mid 0,0)_{x_{0} x_{1} x_{2}}=0, & Z_{\beta_{0}+f}(0 \mid 0,0)_{x_{0} x_{2} x_{2}}=\left(t_{2}-t_{1}\right) \phi^{3}, \\
Z_{\beta_{0}+f}(0 \mid 0,0)_{x_{1} x_{2} x_{2}}=\left(t_{2}-t_{0}\right) \phi^{3}, & Z_{\beta_{0}+f}(0 \mid 0,0)_{x_{0} x_{0} x_{2}}=\left(t_{0}-t_{1}\right) \phi^{3}, \\
Z_{\beta_{0}+f}(0 \mid 0,0)_{x_{1} x_{1} x_{2}}=\left(t_{1}-t_{0}\right) \phi^{3}, & Z_{\beta_{0}+f}(0 \mid 0,0)_{x_{2} x_{2} x_{2}} \\
& =\left(2 t_{2}-t_{0}-t_{1}\right) \phi^{3}, \\
Z_{\beta_{0}+f}(0 \mid 0,0)_{x_{0} x_{1} x_{1}}=\left(t_{1}-t_{2}\right) \phi^{3}, & Z_{\beta_{0}+f}(0 \mid 0,0)_{x_{0} x_{0} x_{1}}=\left(t_{0}-t_{2}\right) \phi^{3}, \\
Z_{\beta_{0}+f}(0 \mid 0,0)_{x_{0} x_{0} x_{0}}=\left(2 t_{0}-t_{1}-t_{2}\right) \phi^{3}, & Z_{\beta_{0}+f}(0 \mid 0,0)_{x_{1} x_{1} x_{1}} \\
& =\left(2 t_{1}-t_{0}-t_{2}\right) \phi^{3} .
\end{array}
$$

Proof. We attach the level $(0,1)$ cap to the level $(0,0)$ pants to obtain the degree $\beta_{0}$, level $(0,1)$ tube. Applying the gluing formula together with Lemmas 3.6 and 3.9 , we get the following relation

$$
\begin{aligned}
& x_{a}(\overbrace{\beta_{0}}^{(0,1)} \supset x_{b}=\overbrace{\beta_{0}-f x_{2}}^{(0,1) x_{2}} \sum_{\beta_{0}+f}^{(0,0)} \int_{x_{a}} x_{b} \\
& Z_{\beta_{0}+f}(0 \mid 0,0)_{x_{2} x_{a} x_{b}}=Z_{\beta_{0}}(0 \mid 0,1)_{x_{a} x_{b}} \phi^{2} .
\end{aligned}
$$

From this, we can get all $Z_{\beta_{0}+f}(0 \mid 0,0)_{x_{a} x_{b} x_{c}}$ with at least one of $a, b, c$ equal to 2 . If we attach the level $(0,-1)$ cap to the level $(0,0)$ pants to obtain the degree $\beta+f$, level $(0,-1)$ tube, we will get

$$
\begin{aligned}
& x_{a}(\sum_{\beta_{0}+f}^{(0,-1)} \supset x_{b}=\overbrace{\beta_{0} x_{0}}^{(0,-1)} \underbrace{1}_{\beta_{0}+f} \int_{x_{a}}^{(0,0)}+\underbrace{(0,-1) x_{x_{1}}^{(1)}}_{\beta_{0}} \underbrace{(0,0)}_{\beta_{0}+f} x_{x_{a}} x_{b} \\
& Z_{\beta_{0}+f}(0 \mid 0,0)_{x_{0} x_{0} x_{1}}-Z_{\beta_{0}+f}(0 \mid 0,0)_{x_{0} x_{1} x_{1}}=\left(t_{0}-t_{1}\right) \phi^{3} \text {, } \\
& Z_{\beta_{0}+f}(0 \mid 0,0)_{x_{0} x_{0} x_{0}}-Z_{\beta_{0}+f}(0 \mid 0,0)_{x_{0} x_{0} x_{1}}=\left(t_{0}-t_{1}\right) \phi^{3} \text {, } \\
& Z_{\beta_{0}+f}(0 \mid 0,0)_{x_{0} x_{1} x_{1}}-Z_{\beta_{0}+f}(0 \mid 0,0)_{x_{1} x_{1} x_{1}}=\left(t_{0}-t_{1}\right) \phi^{3} \text {. }
\end{aligned}
$$


We now write a Frobenius relation as follows (all the pants are level $(0,0)$ ):

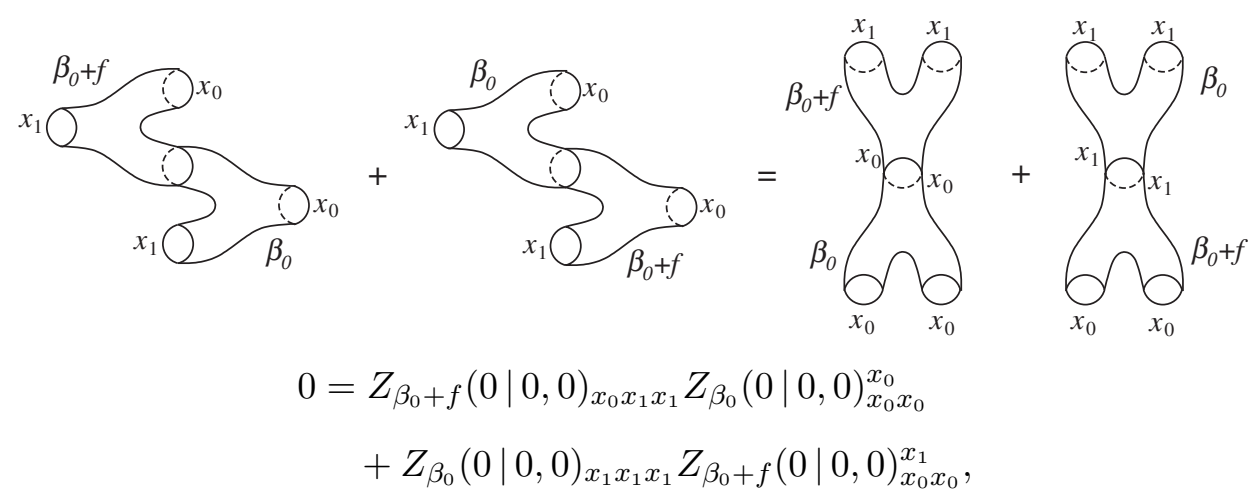

where the left hand side is zero by Lemma 3.4.

By Lemma 3.4, this simplifies to

$$
\left(t_{0}-t_{2}\right) Z_{\beta_{0}+f}(0 \mid 0,0)_{x_{0} x_{1} x_{1}}=\left(t_{1}-t_{2}\right) Z_{\beta_{0}+f}(0 \mid 0,0)_{x_{0} x_{0} x_{1}} .
$$

Combining this with (3.7), we will find the rest of the partition functions in the lemma.

We now know all the partition functions of pants, and so we are able to prove the semi-simplicity of the TQFT (see Remark 2.5):

Proposition 3.11. The level (0,0) TQFT resulted from our setting and Theorem 2.3 is semisimple.

Proof. By Lemmas 3.4 and 3.10, we have

$$
\left.Z(0 \mid 0,0)_{x_{a} x_{b}}^{x_{c}}\right|_{u=0}=Z_{\beta_{0}}(0 \mid 0,0)_{x_{a} x_{b}}^{x_{c}}= \begin{cases}T\left(x_{a}\right) & \text { if } a=b=c \\ 0 & \text { otherwise. }\end{cases}
$$

This means that for $u=0$, the basis $\left\{e_{x_{0}} / T\left(x_{0}\right), e_{x_{1}} / T\left(x_{1}\right), e_{x_{2}} / T\left(x_{2}\right)\right\}$ of the corresponding Frobenius algebra (see Remark 2.5) is idempotent:

$$
\frac{e_{x_{i}}}{T\left(x_{i}\right)} \otimes \frac{e_{x_{j}}}{T\left(x_{j}\right)}=\delta_{i}^{j} \frac{e_{x_{i}}}{T\left(x_{i}\right)}
$$

This proves the semi-simplicity when $u=0$ (see [1], Section 2). Now the proposition follows from Proposition 2.2 in [1]. 


\section{Proof of Theorem 1.6}

We now know everything we need in order to prove Theorem 1.6. We first find the first and second level creation operators and also genus adding operator, which are by definition

$$
U_{1}=\left[Z(0 \mid 1,0)_{x_{a}}^{x_{b}}\right], \quad U_{2}=\left[Z(0 \mid 0,1)_{x_{a}}^{x_{b}}\right], \quad G=\left[Z(1 \mid 0,0)_{x_{a}}^{x_{b}}\right]
$$

respectively. Here partition functions with the lower index $x_{a}$ and the upper index $x_{b}$ are the $(b+1, a+1)$ entry of the matrices above.

We can find $U_{1}$ and $U_{2}$ by simply raising the indices in Lemmas 3.8 and 3.9:

$$
\begin{aligned}
U_{2}= & {\left[Z_{\beta_{0}-f}(0 \mid 0,1)_{x_{a}}^{x_{b}}\right]+\left[Z_{\beta_{0}}(0 \mid 0,1)_{x_{a}}^{x_{b}}\right]=\left[\begin{array}{ccc}
0 & 0 & 0 \\
0 & 0 & 0 \\
0 & 0 & \left(t_{2}-t_{0}\right)\left(t_{2}-t_{1}\right)
\end{array}\right] \phi^{-2} } \\
& +\left[\begin{array}{ccc}
\frac{1}{t_{0}-t_{2}} & 0 & \frac{t_{2}-t_{1}}{\left(t_{0}-t_{1}\right)\left(t_{0}-t_{2}\right)} \\
0 & \frac{1}{t_{1}-t_{2}} & \frac{t_{2}-t_{0}}{\left(t_{1}-t_{0}\right)\left(t_{1}-t_{2}\right)} \\
\frac{1}{t_{2}-t_{0}} & \frac{1}{t_{2}-t_{1}} & \frac{2 t_{2}-t_{0}-t_{1}}{\left(t_{2}-t_{0}\right)\left(t_{2}-t_{1}\right)}
\end{array}\right] \phi
\end{aligned}
$$

$$
=\left[\begin{array}{ccc}
\frac{\phi}{t_{0}-t_{2}} & 0 & \frac{\left(t_{2}-t_{1}\right) \phi}{\left(t_{0}-t_{1}\right)\left(t_{0}-t_{2}\right)} \\
0 & \frac{\phi}{t_{1}-t_{2}} & \frac{\left(t_{2}-t_{0}\right) \phi}{\left(t_{1}-t_{0}\right)\left(t_{1}-t_{2}\right)} \\
\frac{\phi}{t_{2}-t_{0}} & \frac{\phi}{t_{2}-t_{1}} & \frac{\left(t_{2}-t_{0}\right)^{2}\left(t_{2}-t_{1}\right)^{2} \phi^{-2}+\left(2 t_{2}-t_{0}-t_{1}\right) \phi}{\left(t_{2}-t_{0}\right)\left(t_{2}-t_{1}\right)}
\end{array}\right] .
$$

$U_{1}$ is obtained in a similar way, and it is as in Theorem 1.6.

By Lemma 3.4, $\left[Z_{\beta_{0}}(0 \mid 0,0)_{x_{a}}^{x_{b}}\right]$ is the identity matrix, and by the gluing formula, we can write

$$
\left[Z(0 \mid 0,0)_{x_{a}}^{x_{b}}\right]=\left[Z(0 \mid 0,1)_{x_{a}}^{x_{b}}\right]\left[Z(0 \mid 0,-1)_{x_{a}}^{x_{b}}\right]
$$


Therefore, by Lemmas 3.7 and 3.9, we have

$$
\begin{aligned}
U_{2}^{-1}= & {\left[Z_{\beta_{0}}(0 \mid 0,-1)_{x_{a}}^{x_{b}}\right]+\left[Z_{\beta_{0}+f}(0 \mid 0,-1)_{x_{a}}^{x_{b}}\right] } \\
= & {\left[\begin{array}{ccc}
t_{0}-t_{2} & 0 & 0 \\
0 & t_{1}-t_{2} & 0 \\
0 & 0 & 0
\end{array}\right] \phi^{-1} } \\
& +\left[\begin{array}{ccc}
\frac{1}{\left(t_{0}-t_{1}\right)\left(t_{0}-t_{2}\right)} & \frac{1}{\left(t_{0}-t_{1}\right)\left(t_{0}-t_{2}\right)} & \frac{1}{\left(t_{0}-t_{1}\right)\left(t_{0}-t_{2}\right)} \\
\frac{1}{\left(t_{1}-t_{0}\right)\left(t_{1}-t_{2}\right)} & \frac{1}{\left(t_{1}-t_{0}\right)\left(t_{1}-t_{2}\right)} & \frac{1}{\left(t_{1}-t_{0}\right)\left(t_{1}-t_{2}\right)} \\
\frac{1}{\left(t_{2}-t_{0}\right)\left(t_{2}-t_{1}\right)} & \frac{1}{\left(t_{2}-t_{0}\right)\left(t_{2}-t_{1}\right)} & \frac{1}{\left(t_{2}-t_{0}\right)\left(t_{2}-t_{1}\right)}
\end{array}\right] \phi^{2} \\
= & {\left[\begin{array}{cccc}
\frac{\left(t_{0}-t_{1}\right)\left(t_{0}-t_{2}\right)^{2} \phi^{-1}+\phi^{2}}{\left(t_{0}-t_{1}\right)\left(t_{0}-t_{2}\right)} & \frac{\phi^{2}}{\left(t_{0}-t_{1}\right)\left(t_{0}-t_{2}\right)} & \frac{\phi^{2}}{\left(t_{0}-t_{1}\right)\left(t_{0}-t_{2}\right)} \\
\frac{\phi^{2}}{\left(t_{1}-t_{0}\right)\left(t_{1}-t_{2}\right)} & \frac{\left(t_{1}-t_{0}\right)\left(t_{1}-t_{2}\right)^{2} \phi^{-1}+\phi^{2}}{\left(t_{1}-t_{0}\right)\left(t_{1}-t_{2}\right)} & \frac{\phi^{2}}{\left(t_{1}-t_{0}\right)\left(t_{1}-t_{2}\right)} \\
\frac{\phi^{2}}{\left(t_{2}-t_{0}\right)\left(t_{2}-t_{1}\right)} & \frac{\phi^{2}}{\left(t_{2}-t_{0}\right)\left(t_{2}-t_{1}\right)} & \frac{\phi^{2}}{\left(t_{2}-t_{0}\right)\left(t_{2}-t_{1}\right)}
\end{array}\right] . }
\end{aligned}
$$

$U_{1}^{-1}$ is obtained in a similar way. $U_{1}^{-1}$ and $U_{2}^{-1}$ are the first and second level annihilation operators, respectively.

Now we are going to find the matrix $G$. By the same argument as that given for Lemma 3.1, one can prove that

$$
Z(1 \mid 0,0)_{x_{a} x_{b}}=Z_{\beta_{0}}(1 \mid 0,0)_{x_{a} x_{b}}+Z_{\beta_{0}+f}(1 \mid 0,0)_{x_{a} x_{b}}
$$

for $a, b \in\{0,1,2\}$. Thus, we have

$$
G=\left[Z_{\beta_{0}}(1 \mid 0,0)_{x_{a}}^{x_{b}}\right]+\left[Z_{\beta_{0}+f}(1 \mid 0,0)_{x_{a}}^{x_{b}}\right]
$$

For calculating the terms in the right hand side of this, we attach two pants at two points (see the picture) and apply the gluing formula:

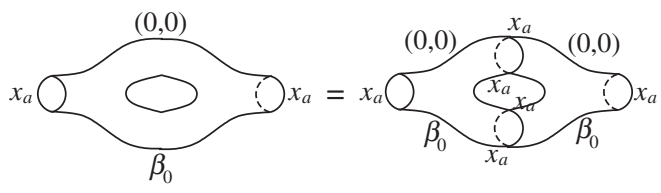




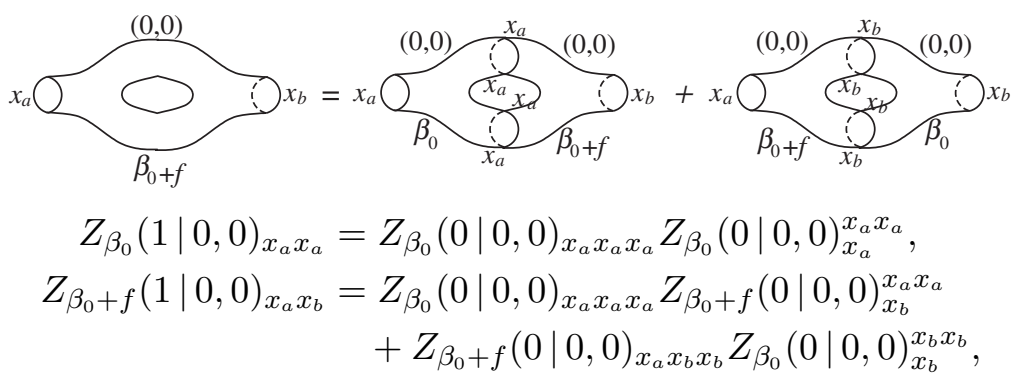

which implies that

$$
\begin{aligned}
& {\left[Z_{\beta_{0}}(1 \mid 0,0)_{x_{a}}^{x_{b}}\right]} \\
& \quad=\left[\begin{array}{ccc}
\left(t_{0}-t_{1}\right)\left(t_{0}-t_{2}\right) & 0 & 0 \\
0 & \left(t_{1}-t_{0}\right)\left(t_{1}-t_{2}\right) & 0 \\
0 & 0 & \left(t_{2}-t_{0}\right)\left(t_{2}-t_{1}\right)
\end{array}\right]
\end{aligned}
$$

and

$$
\begin{aligned}
& {\left[Z_{\beta_{0}+f}(1 \mid 0,0)_{x_{a}}^{x_{b}}\right]} \\
& \quad=\left[\begin{array}{lll}
\frac{2\left(2 t_{0}-t_{1}-t_{2}\right)}{\left(t_{0}-t_{1}\right)\left(t_{0}-t_{2}\right)} & \frac{t_{0}+t_{1}-2 t_{2}}{\left(t_{0}-t_{1}\right)\left(t_{0}-t_{2}\right)} & \frac{t_{0}+t_{2}-2 t_{1}}{\left(t_{0}-t_{1}\right)\left(t_{0}-t_{2}\right)} \\
\frac{t_{0}+t_{1}-2 t_{2}}{\left(t_{1}-t_{0}\right)\left(t_{1}-t_{2}\right)} & \frac{2\left(2 t_{1}-t_{0}-t_{2}\right)}{\left(t_{1}-t_{0}\right)\left(t_{1}-t_{2}\right)} & \frac{t_{1}+t_{2}-2 t_{0}}{\left(t_{1}-t_{0}\right)\left(t_{1}-t_{2}\right)} \\
\frac{t_{0}+t_{2}-2 t_{1}}{\left(t_{2}-t_{0}\right)\left(t_{2}-t_{1}\right)} & \frac{t_{1}+t_{2}-2 t_{0}}{\left(t_{2}-t_{0}\right)\left(t_{2}-t_{1}\right)} & \frac{2\left(2 t_{2}-t_{0}-t_{1}\right)}{\left(t_{2}-t_{0}\right)\left(t_{2}-t_{1}\right)}
\end{array}\right] \phi^{3} .
\end{aligned}
$$

Now Theorem 1.6 for $g \geq 1$ is a direct application of the gluing rules (Theorem 2.3).

The same formula holds for $g=0$, which follows from the semi-simplicity of the level $(0,0)$ TQFT (see Remark 2.5 and Proposition 3.11. See also Section 2 in [1], and Section 5 in [2]).

\section{Proof of Theorem 1.7}

We first prove the following lemma:

Lemma 5.1. Any $\mathbb{P}^{2}$-bundle over a curve $C$ is deformation equivalent to

$$
\mathbb{P}(\mathcal{O} \oplus \mathcal{O} \oplus L) \longrightarrow C
$$

where $\mathcal{O} \rightarrow C$ is the trivial bundle and $L \rightarrow C$ is a line bundle. 
Proof. First, we show that every $\mathbb{P}^{2}$-bundle over a curve $C$ is of the form $\mathbb{P}(E) \rightarrow C$, where $E \rightarrow C$ is a rank 3 bundle. A rank 3 vector bundle (resp. $\mathbb{P}^{2}$-bundle) over $C$ is classified by an element in $\check{H}^{1}(C, \mathrm{Gl}(3)$ ) (resp. $\check{H}^{1}(C, P \mathrm{Gl}(3))$ ), where $\mathrm{Gl}(3)$ (resp. $\left.P \mathrm{Gl}(3)\right)$ is the (non-Abelian) sheaf of $\mathrm{Gl}(3)$ (resp. $P \mathrm{Gl}(3)$ ) valued holomorphic functions on $C$. From the exact sequence of sheaves

$$
0 \longrightarrow \mathcal{O}^{*} \longrightarrow \mathrm{Gl}(3) \longrightarrow P \mathrm{Gl}(3) \longrightarrow 0
$$

we get a map

$$
\check{H}^{1}(C, P \mathrm{Gl}(3)) \longrightarrow \check{H}^{2}\left(C, \mathcal{O}^{*}\right)
$$

By examining the cocycles, one can see that a $\mathbb{P}^{2}$-bundle over $C$ is of the form $\mathbb{P}(E) \rightarrow C$ if and only if the corresponding element in $\check{H}^{1}(C, P \mathrm{Gl}(3))$ goes to zero under the above map. This element is represented by the Cech cocycle obtained from the transition functions of the bundle. But $\check{H}^{2}\left(C, \mathcal{O}^{*}\right)=0$ for a curve $C$; this completes the first part of the proof of the lemma.

Next, we show that $\mathbb{P}(E) \rightarrow C$ is deformation equivalent to

$$
\mathbb{P}(\mathcal{O} \oplus \mathcal{O} \oplus L) \rightarrow C
$$

It is a standard fact that for a rank 3 bundle $E$ over a curve, we have the following exact sequence of bundles over $C$ (see [6], Example 5.0.1):

$$
0 \longrightarrow \mathcal{O} \oplus \mathcal{O} \longrightarrow E(m) \longrightarrow L \longrightarrow 0
$$

for some line bundle $L$ and some $m \gg 0$ such that $E(m)$ is globally generated by its sections. Thus $E(m)$ corresponds to an element

$$
v \in \operatorname{Ext}^{1}(L, \mathcal{O} \oplus \mathcal{O}) .
$$

We can deform $E(m)$ by deforming the extension class $v$ to 0 inside this vector space. But $0 \in \operatorname{Ext}^{1}(L, \mathcal{O} \oplus \mathcal{O})$ corresponds to

$$
\mathcal{O} \oplus \mathcal{O} \oplus L \rightarrow C
$$

So we have proven that $E(m)$ is deformation equivalent to $\mathcal{O} \oplus \mathcal{O} \oplus L$. Now we use the isomorphism $\mathbb{P}(E) \cong \mathbb{P}(E(m))$ to complete the proof of the lemma. 
By this lemma, we assume that the space $X=\mathbb{P}(\mathcal{O} \oplus \mathcal{O} \oplus L)$, and we let $c_{1}(L)=k$. For simplicity, we use the following notations in this section:

$$
\begin{array}{lll}
A=\left[Z_{\beta_{0}}(1 \mid 0,0)_{x_{a}}^{x_{b}}\right], & B=\left[Z_{\beta_{0}+f}(1 \mid 0,0)_{x_{a}}^{x_{b}}\right], \quad C=\left[Z_{\beta_{0}-f}(0 \mid 0,1)_{x_{a}}^{x_{b}}\right], \\
E=\left[Z_{\beta_{0}}(0 \mid 0,1)_{x_{a}}^{x_{b}}\right], \quad N=\left[Z_{\beta_{0}}(0 \mid 0,-1)_{x_{a}}^{x_{b}}\right], \quad M=\left[Z_{\beta_{0}+f}(0 \mid 0,-1)_{x_{a}}^{x_{b}}\right] .
\end{array}
$$

$A$ and $B$ were given at the end of Section 4 , and $C$ and $E$ (respectively, $N$ and $M$ ) were the first and the second matrices in the right hand side of (4.1) (respectively, (4.2)). By using the notations of the previous sections, we can write

$$
G=A+B, \quad U_{2}=C+E, \quad U_{2}^{-1}=N+M,
$$

and hence the formula in Theorem 1.6 (for $k_{1}=0$ and $k_{2}=k$ ) reads as follows:

$$
Z(g \mid 0, k)=\operatorname{tr}\left((A+B)^{g-1}(C+E)^{k}\right) .
$$

Now we are looking for those terms in this formula that correspond to Calabi-Yau section class. If we denote this class by $\beta_{c s}=\beta_{0}+n f$, then $n$ must satisfy

$$
K_{X} \cdot \beta=0 \quad \Longrightarrow \quad 2 g-2-k-3 n=0 .
$$

If for given $g$ and $k$ there is an integral solution for $n$ in this equation, then the Calabi-Yau class exists. We write the above equation in terms of $n$ instead of $k$ :

$$
Z(g \mid 0, k)=\operatorname{tr}\left((A+B)^{g-1}(C+E)^{2 g-2-3 n}\right) .
$$

Now by the gluing formula, $G=A+B$ commutes with $U_{2}=C+E$, and so we have

$$
Z(g \mid 0, k)=\operatorname{tr}\left(\left((A+B)(C+E)^{2}\right)^{g-1}(C+E)^{-3 n}\right)
$$

\subsection{Notation}

For two matrices $U$ and $V$, by $\left(U^{a}, V^{b}\right)$ for $a, b \in \mathbb{Z}^{+}$, we mean the sum of the all the products that we can write containing $a$ copies of $U$ and $b$ copies of $V$. For example

$$
\left(U^{2}, V\right)=U^{2} V+U V U+V U^{2}
$$


We first assume that $g>0$. We distinguish two cases:

(i) $n<0$. One can see that $E^{3}=0$ and $B E^{2}=0$, and so we have

$$
\begin{aligned}
Z(g \mid 0, k)= & \operatorname{tr}\left(\left((A+B)\left(C^{2}+E^{2}+(E, C)\right)\right)^{g-1}\right. \\
& \left.\times\left(\left(E^{2}, C\right)+\left(E, C^{2}\right)+C^{3}\right)^{-n}\right) \\
= & \operatorname{tr}\left(\left(A E^{2}+B(E, C)+\cdots\right)^{g-1}\left(\left(E^{2}, C\right)+\cdots\right)^{-n}\right) .
\end{aligned}
$$

$A, B, C$ and $E$ correspond to the classes $\beta_{0}, \beta_{0}+f, \beta_{0}-f$ and $\beta_{0}$, respectively. One can see that only those terms that have been written in the last equality above contribute to make the class $\beta_{c s}=\beta_{0}+n f$. Thus,

$$
\begin{aligned}
Z_{\beta_{c s}}(g \mid 0, k)= & \operatorname{tr}\left(\left(A E^{2}+B(E, C)\right)^{g-1}\left(\left(E^{2}, C\right)\right)^{-n}\right) \\
= & \operatorname{tr}\left(\left(A E^{2}+B E C+B C E\right)^{g-1}\left(E^{2} C+C E^{2}+E C E\right)^{-n}\right) \\
= & \operatorname{tr}\left(\left(A E^{2}\right)^{g-1}\left(C E^{2}\right)^{-n}\right)+\operatorname{tr}\left((B E C)^{g-1}\left(E^{2} C\right)^{-n}\right) \\
& +\operatorname{tr}\left((B C E)^{g-1}(E C E)^{-n}\right) .
\end{aligned}
$$

For the last equality, we only used the fact that $E^{3}=B E^{2}=0$ again, and also

$$
\operatorname{tr}(U V)=\operatorname{tr}(V U)
$$

for any two matrices $U$ and $V$.

Now one can see easily by induction that for any non-negative integer $a$

$$
A E^{2}\left(C E^{2}\right)^{a}=A E^{2}=\left[\begin{array}{lll}
1 & 1 & 1 \\
1 & 1 & 1 \\
1 & 1 & 1
\end{array}\right] \phi^{2}
$$

Therefore, the first term in (5.2) is

$$
\begin{aligned}
\operatorname{tr}\left(\left(A E^{2}\right)^{g-1}\left(C E^{2}\right)^{-n}\right) & =\operatorname{tr}\left(\left[\begin{array}{lll}
1 & 1 & 1 \\
1 & 1 & 1 \\
1 & 1 & 1
\end{array}\right]^{g-1} \phi^{2 g-2}\right) \\
& =3^{g-1} \phi^{2 g-2}
\end{aligned}
$$


Again induction on non-negative integers $a, b$ together with simple calculations imply that

$$
(B E C)^{b}\left(E^{2} C\right)^{a}=3^{b}\left[\begin{array}{ccc}
0 & 0 & \frac{t_{1}-t_{2}}{t_{0}-t_{2}} \\
0 & 0 & \frac{t_{0}-t_{2}}{t_{1}-t_{0}} \\
0 & 0 & 1
\end{array}\right] \phi^{2 b} .
$$

Therefore, the the second term in (5.2) is

$$
\operatorname{tr}\left((B E C)^{g-1}\left(E^{2} C\right)^{-n}\right)=3^{g-1} \phi^{2 g-2} .
$$

Powers of $B C E$ are more difficult to compute, and so for computing the third term in (5.2) we first notice that

$$
C E B=\left[\begin{array}{lll}
0 & 0 & 0 \\
0 & 0 & 0 \\
3 & 3 & 3
\end{array}\right] \phi^{2},
$$

and also $(E C E)^{a}=E C E$ for any positive integer $a$, and so for $b>1$, we can write

$$
(B C E)^{b}(E C E)^{a}=\left(B(C E B)^{b-1} C E\right)(E C E)=3^{b-1}(B C E)(E C E) \phi^{2 b-2} .
$$

An easy calculation shows that

$$
\operatorname{tr}((B C E)(E C E))=\operatorname{tr}(B C E)=3 \phi^{2} .
$$

Putting all together, we can find the third term in (5.2):

$$
\operatorname{tr}\left((B C E)^{g-1}(E C E)^{-n}\right)=3^{g-1} \phi^{2 g-2} .
$$

By (5.2)-(5.6), we find $Z_{\beta_{c s}}(g \mid 0, k)=3^{g} \phi^{2 g-2}$, which proves the theorem in this case.

(ii) $n \geq 0$. We have $U_{2}^{-1}=M+N$, and so we can rewrite (5.1) as

$$
Z(g \mid 0, k)=\operatorname{tr}\left(\left((A+B)(C+E)^{2}\right)^{g-1}(M+N)^{3 n}\right) .
$$

One can check that $\left(M^{2}, N\right)=M^{3}=0$, and so

$$
Z(g \mid 0, k)=\operatorname{tr}\left(\left(A E^{2}+B(E, C)+\cdots\right)^{g-1}\left(\left(M, N^{2}\right)+N^{3}\right)^{n}\right) .
$$


By the same reason as in the last case, we have

$$
\begin{aligned}
Z_{\beta_{c s}}(g \mid 0, k)= & \operatorname{tr}\left(\left(A E^{2}\right)^{g-1}\left(N^{2} M\right)^{n}\right)+\operatorname{tr}\left((B E C)^{g-1}(N M N)^{n}\right) \\
& +\operatorname{tr}\left((B C E)^{g-1}\left(M N^{2}\right)^{n}\right) .
\end{aligned}
$$

The rest of the proof is similar to the last case and is omitted.

For $g=0$, the result is deduced from the semi-simplicity of the TQFT (Proposition 3.11, see also Remark 2.5).

Remark 5.2. The partition function for a Calabi-Yau section class is a priori independent of the equivariant parameters. Hence, the calculation for Theorem 1.7 can be done with any choice of equivariant parameters. For example, the choice of

$$
t_{0}=0, \quad t_{1}+t_{2}=0
$$

makes the calculations simpler.

\section{Appendix A. Proof of the Gluing theorem}

We first prove the assertion of Remark 2.4, which deals with the fact that we do not need to consider maps with disconnected domains:

Lemma A.1. The contribution of maps with disconnected domain curves in the section class equivariant Gromov-Witten invariants of the space $\mathbb{P}(\mathcal{O} \oplus$ $\left.L_{1} \oplus L_{2}\right)$ is zero.

Proof. A disconnected domain curve whose image represents the class $\beta_{0}+$ $n f$ is a union of a distinguished connected component, whose image represents the class $\beta_{0}+n^{\prime} f$, and a number of other components, whose images represent the class $n^{\prime \prime} f$ for some positive integer $n^{\prime \prime}$. We have

$$
\operatorname{virdim} \bar{M}\left(X / \vec{F}, n^{\prime \prime} f\right)=-\left(-3 H+\left(2 g-2-k_{1}-k_{2}\right) F\right) \cdot n^{\prime \prime} f=3 n^{\prime \prime}>0 .
$$

So by a discussion similar to Remark 2.1, one can see that

$$
\int_{\left[\bar{M}_{h}\left(X / \vec{F}, n^{\prime \prime} f\right)\right]^{\mathrm{vir}}} 1=0 .
$$


Disconnected invariants can be expressed in terms of the products of the connected invariants ${ }^{4}$, and so the lemma follows from the vanishing above.

Now we return to the proof of Theorem 2.3. We prove the first formula, and the proof of the second one is similar. For simplicity, we prove the case $s=0$ and $t=0$. Extending the argument to the general case is straightforward.

Let $C_{0}$ be a connected curve of genus $g$ with two irreducible components, $C^{\prime}$ and $C^{\prime \prime}$ of genera $g^{\prime}$ and $g^{\prime \prime}$ respectively, which are attached together at one point $p$. In other words

$$
C_{0}=C^{\prime} \bigcup_{p=p^{\prime}=p^{\prime \prime}} C^{\prime \prime}
$$

where $p^{\prime} \in C^{\prime}$ and $p^{\prime \prime} \in C^{\prime \prime}$. Now we consider two $\mathbb{P}^{2}$-bundles

$$
X^{\prime}=\mathbb{P}\left(\mathcal{O} \oplus L_{1}^{\prime} \oplus L_{2}^{\prime}\right) \longrightarrow C^{\prime}, \quad X^{\prime \prime}=\mathbb{P}\left(\mathcal{O} \oplus L_{1}^{\prime \prime} \oplus L_{2}^{\prime \prime}\right) \longrightarrow C^{\prime \prime},
$$

where $L_{1}^{\prime}, L_{2}^{\prime}, L_{1}^{\prime \prime}$ and $L_{2}^{\prime \prime}$ are line bundles of degrees $k_{1}^{\prime}, k_{2}^{\prime}, k_{1}^{\prime \prime}$ and $k_{2}^{\prime \prime}$, respectively. We attach these two spaces by identifying the fibers, $F^{\prime}$ and $F^{\prime \prime}$ over $p^{\prime}$ and $p^{\prime \prime}$, respectively, such that the resulting space is $W_{0}=\mathbb{P}(\mathcal{O} \oplus$ $\left.L_{1} \oplus L_{2}\right) \rightarrow C_{0}$, where $L_{1}$ and $L_{2}$ are line bundles of degrees $k_{1}=k_{1}^{\prime}+k_{2}^{\prime}$ and $k_{2}=k_{1}^{\prime \prime}+k_{2}^{\prime \prime}$, respectively. In other words

$$
W_{0}=X^{\prime} \bigcup_{F=F^{\prime}=F^{\prime \prime}} X^{\prime \prime}
$$

where $F$ is the fiber over $p$.

Let $W \rightarrow \mathbb{A}^{1}$ be a generic, 1-parameter deformation of $W_{0}$ for which the fibers $W_{t}$ for $t \neq 0 \in \mathbb{A}^{1}$ are $\mathbb{P}\left(\mathcal{O} \oplus L_{1} \oplus L_{2}\right) \rightarrow C$, where $C$ is a smooth curve of genus $g$, and $L_{1}$ and $L_{2}$ are line bundles of degrees $k_{1}, k_{2}$.

We follow Jun Li's proof of the degeneration formula in [8]. Let $\mathfrak{W}$ be the stack of expanded degenerations of $W$, with central fiber $\mathfrak{W}_{0}$, and let

\footnotetext{
${ }^{4}$ For non-relative invariants, this follows from the elementary fact that the moduli space of disconnected stable maps can easily be expressed in terms of products and finite quotients of connected map moduli spaces. For the relative invariants, the moduli space of disconnected maps no longer has a simple description in terms of the moduli spaces for connected maps; however, the corresponding statement relating the disconnected invariants to the connected invariants still holds. This can be proven using the results of [11], where the authors prove that the relative invariants can always be expressed in terms of the non-relative invariants [13].
} 
$\bar{M}_{h}(\mathfrak{W}, \beta)$ be the stack of non-degenerate, pre-deformable, genus $h$, degree $\beta$ maps to $\mathfrak{W}$, where $\beta$ is a section class [7].

We have the evaluation maps

$$
\mathrm{ev}^{\prime}: \bar{M}_{h^{\prime}}\left(X^{\prime} / F^{\prime}, \beta^{\prime}\right) \longrightarrow F^{\prime}=F, \quad \mathrm{ev}^{\prime \prime}: \bar{M}_{h^{\prime \prime}}\left(X^{\prime} / F^{\prime \prime}, \beta^{\prime \prime}\right) \longrightarrow F^{\prime \prime}=F .
$$

Li constructs a map

$$
\Phi_{\eta}: \bar{M}\left(X^{\prime} / F^{\prime}, \beta^{\prime}\right) \times_{F} \bar{M}\left(X^{\prime \prime} / F^{\prime \prime}, \beta^{\prime \prime}\right) \rightarrow \bar{M}\left(\mathfrak{W}_{0}, \beta\right),
$$

where $\eta$ includes a pair of classes $\left(\beta^{\prime}, \beta^{\prime \prime}\right)$, such that $\beta=\beta^{\prime}+\beta^{\prime \prime}$ and a pair of genera $\left(h^{\prime}, h^{\prime \prime}\right)$, such that $h=h^{\prime}+h^{\prime \prime}$. Then, he gives a virtual cycle formula, which in our case is

$$
\left[\bar{M}\left(\mathfrak{W}_{0}\right), \beta\right]^{\mathrm{vir}}=\sum_{\eta} \Phi_{\eta, *} \Delta^{!}\left(\left[\bar{M}\left(X^{\prime} / F^{\prime}, \beta^{\prime}\right)\right]^{\mathrm{vir}} \times\left[\bar{M}\left(X^{\prime \prime} / F^{\prime \prime}, \beta^{\prime \prime}\right)\right]^{\mathrm{vir}}\right),
$$

where $\Delta: F \rightarrow F \times F$ is the diagonal map.

Remark A.2. The torus action on the family $W \rightarrow \mathbb{A}^{1}$ gives an action on the stack of expanded degeneration, $\mathfrak{W}$. One can check that pre-deformability condition is invariant under this action, and so it induces (canonically) an action on each of the moduli spaces $\bar{M}_{h^{\prime}}\left(X^{\prime} / F^{\prime}, \beta^{\prime}\right), \bar{M}_{h^{\prime \prime}}\left(X^{\prime \prime} / F^{\prime \prime}, \beta^{\prime \prime}\right)$ and $\bar{M}_{h}(\mathfrak{W}, \beta)$. Therefore, Li's formula holds in the equivariant Chow groups.

If we work with the basis elements $x_{0}, x_{1}, x_{2}$ (introduced in Section 2), for the equivariant Chow group of the fiber $F$, by using (2.2), one can see easily that

$$
x_{0}^{\vee}=\frac{x_{0}}{T\left(x_{0}\right)}, \quad x_{1}^{\vee}=\frac{x_{1}}{T\left(x_{1}\right)}, \quad x_{2}^{\vee}=\frac{x_{2}}{T\left(x_{2}\right)}
$$

is its dual basis, and so the cohomology class of the diagonal of $F \times F$ is given by $[12$, Theorem 11.11]

$$
\begin{aligned}
\operatorname{im}(\Delta) & =\sum_{i=0}^{2} x_{i} \times x_{i}^{\vee} \\
& =x_{0} \times \frac{x_{0}}{T\left(x_{0}\right)}+x_{1} \times \frac{x_{1}}{T\left(x_{1}\right)}+x_{2} \times \frac{x_{2}}{T\left(x_{2}\right)} .
\end{aligned}
$$


Using this, we can rewrite (A.1) as

$$
\begin{aligned}
{\left[\bar{M}\left(\mathfrak{W}_{0}, \beta\right)\right]^{\mathrm{vir}}=} & \sum_{\eta} \Phi_{\eta, *}\left(\sum_{i=0}^{2}\left(\mathrm{ev}^{\prime}\right)^{*}\left(x_{i}\right) \cap\left[\bar{M}\left(X^{\prime} / F^{\prime}, \beta^{\prime}\right)\right]^{\mathrm{vir}}\right. \\
& \left.\times \frac{\left(\mathrm{ev}^{\prime \prime}\right)^{*}\left(x_{i}\right)}{T\left(x_{i}\right)} \cap\left[\bar{M}\left(X^{\prime \prime} / F^{\prime \prime}, \beta^{\prime \prime}\right)\right]^{\mathrm{vir}}\right) .
\end{aligned}
$$

We now have

$$
\begin{aligned}
Z_{\beta}^{h}\left(g \mid k_{1}, k_{2}\right) & =\int_{\left[\bar{M}\left(\mathfrak{W}_{t}, \beta\right)\right]^{\mathrm{vir}}} 1=\int_{\left[\bar{M}\left(\mathfrak{W}_{0}, \beta\right)\right]^{\mathrm{vir}}} 1 \\
& =\sum_{\eta} \sum_{i=0}^{2} \int_{\left[\bar{M}\left(X^{\prime} / F^{\prime}, \beta^{\prime}\right)\right] \mathrm{vir}^{\mathrm{vir}}}\left(\mathrm{ev}^{\prime}\right)^{*}\left(x_{i}\right) \int_{\left[\bar{M}\left(X^{\prime \prime} / F^{\prime \prime}, \beta^{\prime \prime}\right)\right]^{\mathrm{vir}}} \frac{\left(\mathrm{ev}^{\prime \prime}\right)^{*}\left(x_{i}\right)}{T\left(x_{i}\right)} \\
& =\sum_{\eta} \sum_{i=0}^{2} Z_{\beta^{\prime}}^{h^{\prime}}\left(g^{\prime} \mid k_{1}^{\prime}, k_{2}^{\prime}\right)_{x_{i}} Z_{\beta^{\prime \prime}}^{h^{\prime \prime}}\left(g^{\prime \prime} \mid k_{1}^{\prime \prime}, k_{2}^{\prime \prime}\right)^{x_{i}} .
\end{aligned}
$$

Then we can write

$$
\begin{aligned}
Z\left(g \mid k_{1}, k_{2}\right)= & \sum_{\beta \text { is a section class }} \sum_{h} u^{2 h-2-K_{X} \cdot \beta} Z_{\beta}^{h}\left(g \mid k_{1}, k_{2}\right) \\
= & \sum_{\beta} \sum_{h} u^{2 h-2-K_{X} \cdot \beta} \sum_{\eta} \sum_{i=0}^{2} Z_{\beta^{\prime}}^{h^{\prime}}\left(g^{\prime} \mid k_{1}^{\prime}, k_{2}^{\prime}\right)_{x_{i}} Z_{\beta^{\prime \prime}}^{h^{\prime \prime}}\left(g^{\prime \prime} \mid k_{1}^{\prime \prime}, k_{2}^{\prime \prime}\right)^{x_{i}} \\
= & \sum_{\beta, h, \eta, i} u^{2 h^{\prime}-2-K_{X^{\prime}} \cdot \beta^{\prime}} Z_{\beta^{\prime}}^{h^{\prime}}\left(g^{\prime} \mid k_{1}^{\prime}, k_{2}^{\prime}\right)_{x_{i}} u^{2 h^{\prime \prime}-2-K_{X^{\prime \prime}} \cdot \beta^{\prime \prime}} \\
& \cdot Z_{\beta^{\prime \prime}}^{h^{\prime \prime}}\left(g^{\prime \prime} \mid k_{1}^{\prime \prime}, k_{2}^{\prime \prime}\right)^{x_{i}} \\
= & \sum_{i=0}^{2} Z\left(g^{\prime} \mid k_{1}^{\prime}, k_{2}^{\prime}\right)_{x_{i}} Z\left(g^{\prime \prime} \mid k_{1}^{\prime \prime}, k_{2}^{\prime \prime}\right)^{x_{i}} .
\end{aligned}
$$

\section{Appendix B. Some special cases}

In this appendix, we provide a few more applications of Theorem 1.6. The proofs of the first three theorems are straightforward. One first expands the formula in Theorem 1.6 in each case to get a polynomial in $t_{0}, t_{1}$ and $t_{2}$. The partition function that appeared in the statement of each theorem then corresponds to the terms in this polynomial of a specific degree, determined by the given curve class in that theorem (see Remark 2.1, and also the proofs of Theorem 1.7 and Theorem B.4). 
Theorem B.1. Assume that $k_{1}>0$ and $k_{2} \geq 0$. Then the degree $\beta_{0}-$ $k_{1} f$, level $\left(k_{1},-k_{2}\right)$ equivariant Gromov-Witten partition function of $X$ is given by

$$
Z_{\beta_{0}-k_{1} f}\left(g \mid k_{1},-k_{2}\right)=\left(t_{1}-t_{0}\right)^{g+k_{1}-1}\left(t_{1}-t_{2}\right)^{g+k_{1}+k_{2}-1}\left(2 \sin \frac{u}{2}\right)^{-2 k_{1}-k_{2}} .
$$

Theorem B.2. Assume that $k>0$. Then the degree $\beta_{0}-k f$, level $(k, k)$ equivariant Gromov-Witten partition function of $X$ is given by

$$
\begin{aligned}
Z_{\beta_{0}-k f}(g \mid k, k)= & \left(\left(t_{1}-t_{0}\right)^{g+k-1}\left(t_{1}-t_{2}\right)^{g-1}\right. \\
& \left.+\left(t_{2}-t_{0}\right)^{g+k-1}\left(t_{2}-t_{1}\right)^{g-1}\right)\left(2 \sin \frac{u}{2}\right)^{-k}
\end{aligned}
$$

Theorem B.3. Assume that $k_{1} \geq 0$ and $k_{2} \geq 0$. Then the degree $\beta_{0}$, level $\left(-k_{1},-k_{2}\right)$ equivariant Gromov-Witten partition function of $X$ is given by

$$
\begin{aligned}
Z_{\beta_{0}}(g \mid- & \left.k_{1},-k_{2}\right) \\
= & \begin{cases}\left(t_{0}-t_{1}\right)^{g+k_{1}-1}\left(t_{0}-t_{2}\right)^{g+k_{2}-1}\left(2 \sin \frac{u}{2}\right)^{k_{1}+k_{2}} & k_{1}>0, \quad k_{2}>0 \\
\left(\left(t_{0}-t_{1}\right)^{g+k_{1}-1}\left(t_{0}-t_{2}\right)^{g-1}\right. & \\
\left.+\left(t_{2}-t_{0}\right)^{g-1}\left(t_{2}-t_{1}\right)^{g+k_{1}-1}\right)\left(2 \sin \frac{u}{2}\right)^{k_{1}} & k_{1}>0, \quad k_{2}=0, \\
\left(\left(t_{0}-t_{1}\right)^{g-1}\left(t_{0}-t_{2}\right)^{g+k_{2}-1}\right. & \\
\left.+\left(t_{1}-t_{0}\right)^{g-1}\left(t_{1}-t_{2}\right)^{g+k_{2}-1}\right)\left(2 \sin \frac{u}{2}\right)^{k_{2}} & k_{1}=0, \quad k_{2}>0 \\
\left(t_{0}-t_{1}\right)^{g-1}\left(t_{0}-t_{2}\right)^{g-1}+\left(t_{1}-t_{0}\right)^{g-1} & \\
\left(t_{1}-t_{2}\right)^{g-1}+\left(t_{2}-t_{0}\right)^{g-1}\left(t_{2}-t_{1}\right)^{g-1} & k_{1}=0, \quad k_{2}=0 .\end{cases}
\end{aligned}
$$

Theorem B.4. Let $n$ be the greatest integer that satisfies $3 n \leq 2 g-2$. Then the degree $\beta_{0}+n f$, level $(0,0)$ equivariant Gromov-Witten partition function of $X$ is given by

$$
Z_{\beta_{0}+n f}(g \mid 0,0)= \begin{cases}0 & g=3 k, \\ 3^{g}\left(2 \sin \frac{u}{2}\right)^{2 g-2} & g=3 k+1 \\ 3^{g-2}(g-1)\left(t_{0}^{2}+t_{1}^{2}+t_{2}^{2}\right. & \\ \left.-t_{0} t_{1}-t_{0} t_{2}-t_{1} t_{2}\right)\left(2 \sin \frac{u}{2}\right)^{2 g-4} & g=3 k+2 .\end{cases}
$$


Proof. The case $g=0$ again follows from the semi-simplicity of the level $(0,0)$ TQFT (Corollary 3.2.5, see also Remark 2.5), once one knows the result for $g>0$. So we will assume that $g>0$.

Applying Theorem 1.6 to this case, we can write

$$
Z(g \mid 0,0)=\operatorname{tr}\left((A+B)^{g-1}\right),
$$

where $A$ and $B$ were defined in Section 5 . We prove each case in this theorem separately:

(i) $g=3 k$. In this case, $n=2 k-1$, and one can see that (by using the notation introduced in Section 5):

$$
Z_{\beta_{0}+(2 k-1) f}(g \mid 0,0)=\operatorname{tr}\left(\left(A^{k}, B^{2 k-1}\right)\right) .
$$

We have

$$
A B^{2}=\left[\begin{array}{lll}
1 & 1 & 1 \\
1 & 1 & 1 \\
1 & 1 & 1
\end{array}\right] 9 \phi^{6},
$$

so for any positive integer $a$, we have

$$
\left(A B^{2}\right)^{a}=3^{3 a-3} \phi^{6 a-6} A B^{2} .
$$

One can prove easily that

$$
B^{3}=0, \quad\left(A B A B^{2}\right)^{2}=0 .
$$

Applying (5.3) a few times to each term of $\operatorname{tr}\left(\left(A^{k}, B^{2 k-1}\right)\right)$, and using the other equalities above, we can prove that each of these terms is either zero or is equal to

$$
\operatorname{tr}\left(A B\left(A B^{2}\right)^{k-1}\right)=3^{3 k-6} \phi^{6 k-12} \operatorname{tr}\left(A B A B^{2}\right) .
$$

However, an easy calculation shows that $\operatorname{tr}\left(A B A B^{2}\right)=0$. This proves that each term of $\operatorname{tr}\left(\left(A^{k}, B^{2 k-1}\right)\right)$ is zero. So,

$$
Z_{\beta_{0}+(2 k-1) f}(g \mid 0,0)=0 .
$$

(ii) $g=3 k+1$. In this case $\beta_{0}+n f$ is the Calabi-Yau section class, and we have proved the theorem for this case in more generality in Section 5 . 
(iii) $g=3 k+2$. In this case $n=2 k$, and this time we have

$$
Z_{\beta_{0}+2 k f}(g \mid 0,0)=\operatorname{tr}\left(\left(A^{k+1}, B^{2 k}\right)\right) .
$$

The cases $k=0,1$ can be proved by easy calculations, and so we assume that $k>1$. Applying (5.3) a few times to each term of this, and using (B.2), we can prove that each term of $\operatorname{tr}\left(\left(A^{k+1}, B^{2 k}\right)\right)$ is either zero or is equal to either of

$$
\operatorname{tr}\left(A\left(A B^{2}\right)^{k}\right), \quad \operatorname{tr}\left((A B)^{2}\left(A B^{2}\right)^{k-1}\right), \quad \operatorname{tr}\left(A^{2} B^{2}\left(A B^{2}\right)^{k-1}\right),
$$

where the number of the terms of the first, the second and the third kinds are $2 k+1,3 k+1$ and $k$, respectively. So we can write

$$
Z_{\beta_{0}+2 k f}(g \mid 0,0)=(2 k+1) \operatorname{tr}\left(A\left(A B^{2}\right)^{k}\right)+(3 k+1) \operatorname{tr}\left((A B)^{2}\left(A B^{2}\right)^{k-1}\right)
$$

$$
+k \operatorname{tr}\left(A^{2} B^{2}\left(A B^{2}\right)^{k-1}\right) \text {. }
$$

Using (B.1), we can write

$$
\begin{aligned}
\operatorname{tr}\left(A\left(A B^{2}\right)^{k}\right) & =3^{3 k-3} \phi^{6 k-6} \operatorname{tr}\left(A\left(A B^{2}\right)\right) \\
& =3^{3 k-1}\left(t_{0}^{2}+t_{1}^{2}+t_{2}^{2}-t_{0} t_{1}-t_{0} t_{2}-t_{1} t_{2}\right) \phi^{6 k} \\
\operatorname{tr}\left((A B)^{2}\left(A B^{2}\right)^{k-1}\right) & =3^{3 k-6} \phi^{6 k-12} \operatorname{tr}\left((A B)^{2}\left(A B^{2}\right)\right) \\
& =2 \cdot 3^{3 k-1}\left(t_{0}^{2}+t_{1}^{2}+t_{2}^{2}-t_{0} t_{1}-t_{0} t_{2}-t_{1} t_{2}\right) \phi^{6 k} \\
\operatorname{tr}\left(A^{2} B^{2}\left(A B^{2}\right)^{k-1}\right) & =3^{3 k-6} \phi^{6 k-12} \operatorname{tr}\left(A^{2} B^{2}\left(A B^{2}\right)\right) \\
& =3^{3 k-1}\left(t_{0}^{2}+t_{1}^{2}+t_{2}^{2}-t_{0} t_{1}-t_{0} t_{2}-t_{1} t_{2}\right) \phi^{6 k}
\end{aligned}
$$

We define $Q:=t_{0}^{2}+t_{1}^{2}+t_{2}^{2}-t_{0} t_{1}-t_{0} t_{2}-t_{1} t_{2}$. Putting all these into (B.3), we get

$$
\begin{aligned}
Z_{\beta}(g \mid 0,0) & =3^{3 k-1}(2 k+1+2(3 k+1)+k) Q \phi^{6 k} \\
& =3^{3 k}(3 k+1) Q \phi^{6 k}=3^{g-2}(g-1) Q \phi^{2 g-4},
\end{aligned}
$$

and this proves the theorem in this case. 


\section{Acknowledgment}

I would like to express deep gratitude to my supervisor Jim Bryan whose guidance was crucial for the successful completion of this work. I would also like to thank T. Graber and R. Pandharipande for useful discussions and comments.

\section{References}

[1] J. Bryan and R. Pandharipande, Curves in Calabi-Yau 3-folds and topological quantum field theory, Duke Math. J., Preprint version: math.AG/0306316.

[2] J. Bryan and R. Pandharipande, The local Gromov-Witten theory of curves, Preprint math.AG/0411037, 2004.

[3] A. Gholampour and Y. Song, Evidence for the Gromov-Witten/ Donaldoson-Thomas correspondence, Preprint math.AG/0510006. Math. Res. Lett., 13(4) (2006), 623-629.

[4] T. Graber, Personal communication.

[5] T. Graber and R. Vakil, Relative virtual localization and vanishing of tautological classes on moduli spaces of curves, arXiv: math.AG/0309227.

[6] D. Huybrechts and M. Lehn, The Geometry of Moduli Spaces of Sheaves, Aspects of Mathematics, E31. Friedr. Vieweg \& Sohn, Braunschweig, 1997.

[7] J. Li, Stable morphisms to singular schemes and relative stable morphisms, J. Diff. Geom., 57(3) (2001), 509-578, arXiv: math.AG/0009097.

[8] J. Li, A degeneration formula of $G W$-invariants. J. Diff. Geom., 60(2), (2002), 199-293.

[9] D. Maulik, N. Nekrasov, A. Okounkov and R. Pandharipande, Gromov-Witten theory and Donaldson-Thomas theory, I, ITEP-TH61/03, IHES/M/03/67, arXiv:math.AG/0312059.

[10] D. Maulik, N. Nekrasov, A. Okounkov and R. Pandharipande, Gromov-Witten theory and Donaldson-Thomas theory, II, ITEP-TH61/03, IHES/M/03/67, arXiv:math.AG/0406092. 
[11] D. Maulik and R. Pandharipande, A topological view of GromovWitten theory, arXiv:math.AG/0412503.

[12] J. W. Milnor and J. D. Stasheff, Characteristic Classes, Princeton University Press, Princeton, NJ, 1974, Ann. Math. Stud., no. 76.

[13] R. Pandharipande, Personal communication.

Department of Mathematics

UNIVERSiTy of British COlumbia

1984 MaTHEMATICS ROAD

VANCOUVER, B.C.

CANAdA V6T $1 \mathrm{Z} 2$

E-mail address: amin@math.ubc.ca

ReCeived February 2, 2006 
